
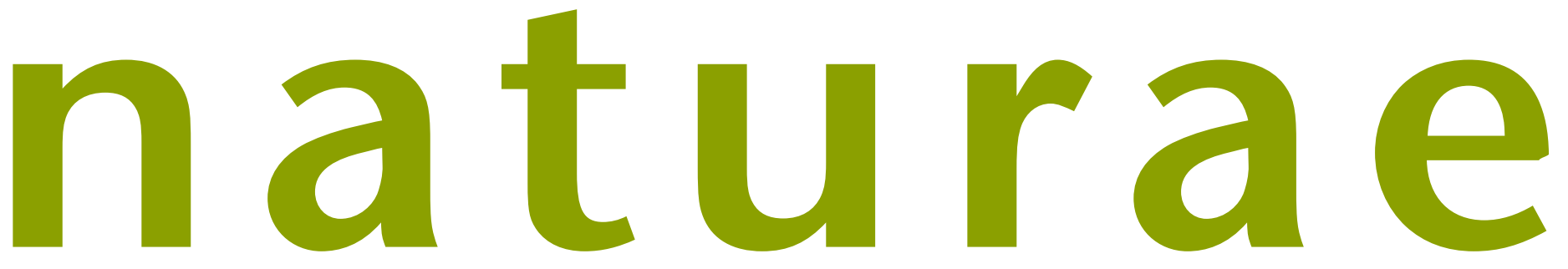

$2021 \cdot 1$

Comparaison de la biodiversité floristique entre berge et île de Loire. Étude de cas dans la réserve naturelle nationale de Saint-Mesmin (45)

Richard CHEVALIER, Michel CHANTEREAU, Rémi DUPRÉ, André EVETTE, Sabine GREULICH, Damien HÉMERAY, Anders MÅRELL, Hilaire MARTIN \& Marc VILLAR

4. ant 2021 (1) - publié le 6. Whart.2021 (1) - Públiét
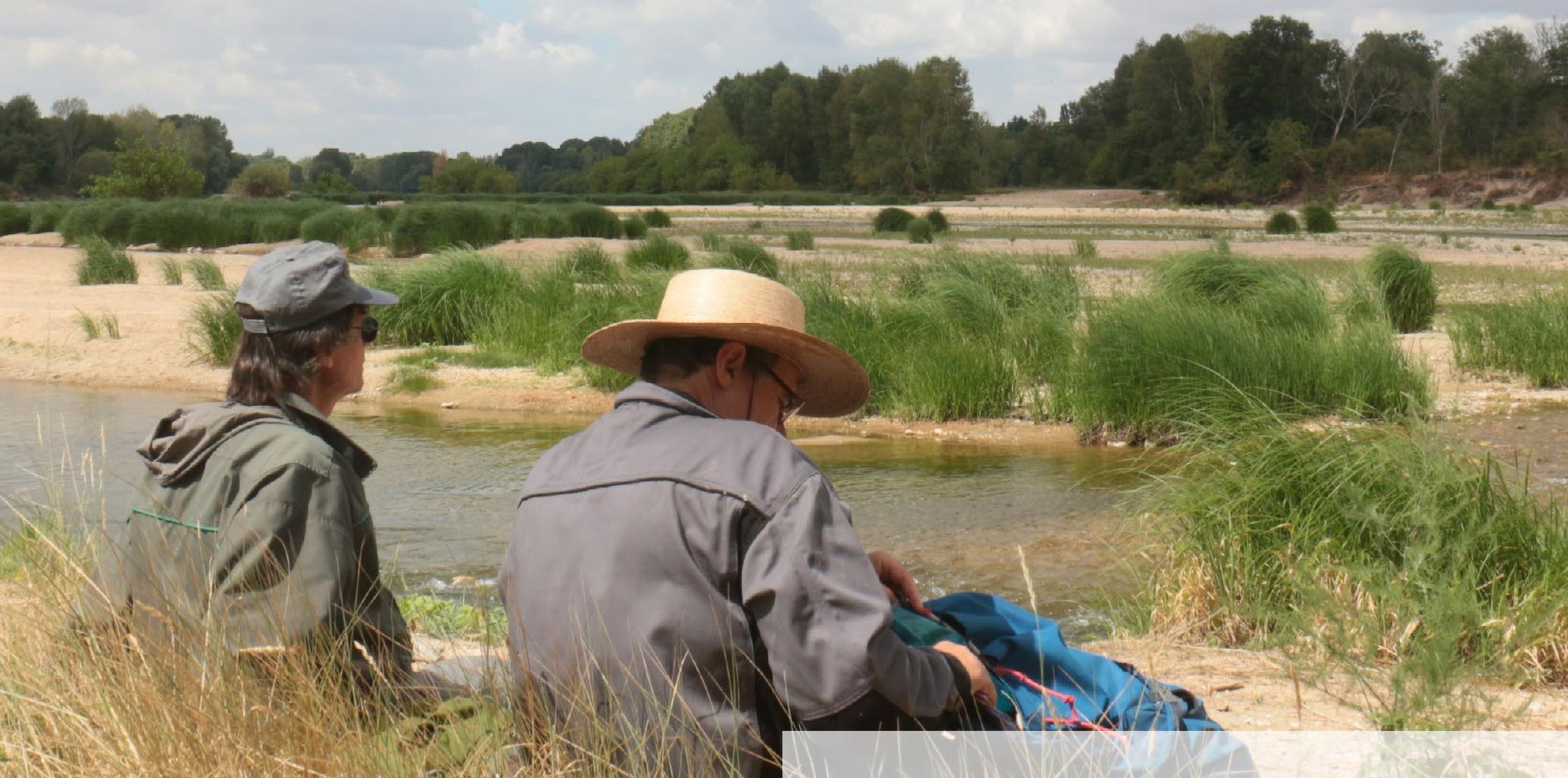

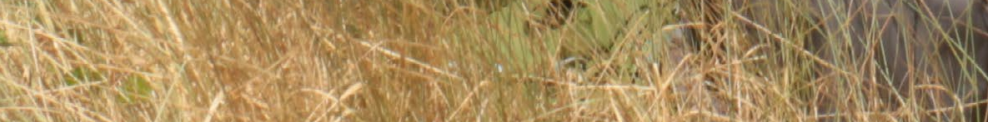

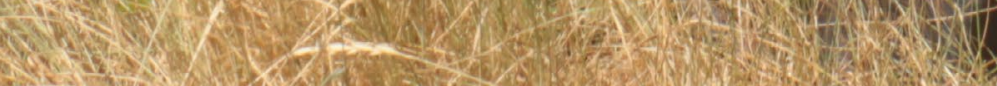
(st) 
DiRECTEUR DE LA PUBLICATION / PUBLICATION DIRECTOR: Bruno David,

Président du Muséum national d'Histoire naturelle

RÉDACTEUR EN CHEF / EDITOR-IN-CHIEF: Jean-Philippe Siblet

ASSISTANTE DE RÉDACTION / ASSISTANT EDITOR: Sarah Figuet (naturae@mnhn.fr)

Mise EN PAGE / PAGE LAYOUT: Sarah Figuet

COMITÉ SCIENTIFIQUE / SCIENTIFIC BOARD:

Luc Abbadie (UPMC, Paris)

Luc Barbier (Parc naturel régional des caps et marais d'Opale, Colembert)

Aurélien Besnard (CEFE, Montpellier)

Vincent Boullet (Expert indépendant flore/végétation, Frugières-le-Pin)

Hervé Brustel (École d'ingénieurs de Purpan, Toulouse)

Patrick De Wever (MNHN, Paris)

Thierry Dutoit (UMR CNRS IMBE, Avignon)

Éric Feunteun (MNHN, Dinard)

Romain Garrouste (MNHN, Paris)

Grégoire Gautier (DRAAF Occitanie, Toulouse)

Olivier Gilg (Réserves naturelles de France, Dijon)

Frédéric Gosselin (Irstea, Nogent-sur-Vernisson)

Patrick Haffner (UMS PatriNat, Paris)

Frédéric Hendoux (MNHN, Paris)

Xavier Houard (OPIE, Guyancourt)

Isabelle Le Viol (MNHN, Concarneau)

Francis Meunier (Conservatoire d'espaces naturels - Hauts-de-France, Amiens)

Serge Muller (MNHN, Paris)

Francis Olivereau (DREAL Centre, Orléans)

Laurent Poncet (UMS PatriNat, Paris)

Nicolas Poulet (OFB, Vincennes)

Jean-Philippe Siblet (UMS PatriNat, Paris)

Laurent Tillon (ONF, Paris)

Julien Touroult (UMS PatriNat, Paris)

COUVERTURE / COVER:

Vue des îles de Mareau-aux-Prés (45) depuis la berge. Crédit photo: Rémi Dupré.

Naturae est une revue en flux continu publiée par les Publications scientifiques du Muséum, Paris Naturae is a fast track journal published by the Museum Science Press, Paris

Les Publications scientifiques du Muséum publient aussi / The Museum Science Press also publish:

Adansonia, Zoosystema, Anthropozoologica, European Journal of Taxonomy, Geodiversitas, Cryptogamie sous-sections Algologie, Bryologie, Mycologie, Comptes Rendus Palevol.

Diffusion - Publications scientifiques Muséum national d'Histoire naturelle

CP $41-57$ rue Cuvier F-75231 Paris cedex 05 (France)

Tél.: 33 (0)1 40794805 / Fax: 33 (0)1 40793840

diff.pub@mnhn.fr / http://sciencepress.mnhn.fr

(C) Publications scientifiques du Muséum national d'Histoire naturelle, Paris, 2021

ISSN (électronique / electronic): 1638-9387 


\title{
Comparaison de la biodiversité floristique entre berge et île de Loire. Étude de cas dans la réserve naturelle nationale de Saint-Mesmin (45)
}

\author{
Richard CHEVALIER \\ Institut national de recherche pour l'agriculture, l'alimentation et l'environnement (INRAE), \\ Domaine des Barres, F-45290 Nogent-sur-Vernisson (France) \\ richard.chevalier@inrae.fr \\ Michel CHANTEREAU \\ Loiret Nature Environnement, 64 route d'Olivet, F-45100 Orléans (France) \\ michel.chantereau@orange.fr
}

Rémi DUPRÉ

Conservatoire botanique national du Bassin parisien (CBNBP), 5 avenue Buffon, F-45064 Orléans (France)

remi.dupre@mnhn.fr

André EVETTE

Université Grenoble Alpes, Institut national de recherche pour l'agriculture, l'alimentation et l'environnement (INRAE), 2 rue de la Papeterie, F-38402 St-Martin-d'Hères (France) andre.evette@inrae.fr

Sabine GREULICH

Université François Rabelais, 33 Allée Ferdinand de Lesseps, F-37204 Tours Cedex (France) greulich@univ-tours.fr

Damien HÉMERAY

Loiret Nature Environnement, 64 route d'Olivet, F-45100 Orléans (France) damien.hemeray@espaces-naturels.fr

Anders MÅRELL

Hilaire MARTIN

Institut national de recherche pour l'agriculture, l'alimentation et l'environnement (INRAE),

Domaine des Barres, F-45290 Nogent-sur-Vernisson (France) anders.marell@inrae.fr hilaire.martin@inrae.fr

Marc VILLAR

Institut national de recherche pour l'agriculture, l'alimentation et l'environnement (INRAE), 2163 Avenue de la Pomme de Pin, CS 40001 Ardon, F-45075 Orléans Cedex 2 (France) marc.villar@inrae.fr

Soumis le 17 février 2020 | Accepté le 21 août 2020 | Publié le 6 janvier 2021

Chevalier R., Chantereau M., Dupré R., Evette A., Greulich S., Hémeray D., Mårell A., Martin H. \& Villar M. 2021. Comparaison de la biodiversité floristique entre berge et île de Loire. Étude de cas dans la réserve naturelle nationale de Saint-Mesmin (45). Naturae 2021 (1): 1-20. https://doi.org/10.5852/naturae2021a1 
MOTS CLÉS

Ripisylve, flore vasculaire espèce patrimoniale, espèce invasive,

grève, eutrophisation, anthropisation, urbanisation.

KEY WORDS

Riparian forest, vascular flora, species of conservation interest,

invasive species, beach, forest, eutrophication, anthropization, urbanisation.

\section{RÉSUMÉ}

Menée au sein de la réserve naturelle nationale (RNN) de Saint-Mesmin (45), sur la Loire, en périphérie aval de la ville d'Orléans, cette étude compare la biodiversité floristique de la ripisylve d'une berge à celle de deux îles boisées en vis-à-vis. L'hypothèse est que la berge, davantage soumise à la pression anthropique, présente une biodiversité altérée. Les relevés floristiques ont été effectués à l'échelle de l'écocomplexe (environ deux hectares par entité), en trois passages annuels et pendant quatre années (2013 à 2016). La berge et chaque île ont une richesse totale similaire, d'environ 310 espèces, et présentent des différences de composition modérées. La principale différence concerne une plus forte présence des espèces oligotrophiles, localisées aux grèves et pelouses des îles, alors que les espèces eutrophiles sont favorisées sur la berge. Si les espèces patrimoniales, toujours en faible effectif, ont tendance à être plus nombreuses sur les îles, la berge en accueille aussi de façon complémentaire. Les espèces exogènes ne sont pas plus nombreuses sur la berge que sur les îles alors que les espèces invasives sont plus abondantes sur les îles. Au final, les ripisylves de berge présentent une flore aussi intéressante que celle des îles, leur composition est complémentaire au bénéfice de la biodiversité de la RNN. Les berges boisées méritent d'être gérées avec attention du fait de leur forte exposition à la fréquentation humaine dans ce contexte péri-urbain, ce qui demande notamment de sensibiliser les promeneurs à leur fragilité. Si cette étude de cas est utile pour la gestion de la RNN, la généralisation des résultats nécessiterait l'examen préalable d'autres couples berge/île le long de la Loire.

\begin{abstract}
Comparing riverbank and island floristic biodiversity on the Loire River. Case study in Saint-Mesmin National Nature Reserve (45).

We compared the floristic biodiversity on two wooded islands with the biodiversity in the riparian forest along the nearby riverbank. Our hypothesis was that the riverbank, which undergoes more anthropic pressure, would host a more altered biodiversity. We carried out floristic surveys within the National Nature Reserve (RNN) of Saint-Mesmin (France), on the Loire, slightly downstream from the city of Orléans. Measurements were taken on areas of about two ha, three times per year from 2013 to 2016. The riverbank and each island had almost the same total species richness (about 310). However, there were moderate differences in composition. First, there were more oligotrophic species on the island beaches and grasslands, and more eutrophic species on the bank. Second, we found slightly more species of conservation interest on the islands, even though the number of individuals was low. The riverbank complements this biodiversity with a few other species. Third, there were not as many non-native species on the riverbank as on the islands but invasive non-natives were more abundant on the islands. Finally, riparian both on the riverbank and on the islands contribute to the biodiversity of the RNN. Wooded riverbanks are more exposed to human activity in this peri-urban context and should be carefully managed. Visitors should be made aware of the value of these areas. While this case study is useful for the management of the RNN, the generalisation of the results would require the observation of other riverbank/island pairs along the Loire.
\end{abstract}

\section{INTRODUCTION}

En tant qu'écotone entre milieux aquatiques et terrestres, les ripisylves ou cordons rivulaires constituent des reliques de la forêt alluviale qui a régressé face aux activités humaines. Ces écosystèmes linéaires assurent de multiples services (qualité du paysage, stabilisation de la berge, régulation des crues, filtre pour la qualité de l'eau, refuge pour la biodiversité) (Naiman \& Décamps 1997) et méritent d'être étudiés et préservés au sein des paysages anthropisés (Claessens et al. 2009). Bien qu'ils occupent une faible part du paysage, les milieux ripariens contribuent significativement à la richesse spécifique régionale (Sabo et al. 2005) et tout particulièrement pour les communautés végétales associées (Nilsson \& Svedmark 2002; Nobis et al. 2016).

Comme toutes les grandes vallées françaises, celle de la Loire est depuis longtemps affectée par les activités humaines. Malgré cela, sa valeur écologique demeure exceptionnelle. Parmi les grands fleuves français, la Loire compte la plus grande longueur et la plus forte proportion de linéaire simultanément concernée par la Directive "Oiseaux» (Zone de Protection Spéciale $=$ ZPS) et la Directive «Habitats, Faune, Flore» (Zone Spéciale de Conservation = ZSC) du réseau Natura 2000 français (Direction générale de l'aménagement, du logement et de la nature 2016). Sur ces habitats ligériens à forte valeur écologique, s'exprime une flore remarquable (Boudin et al. 2007). La Loire moyenne cumule ainsi une forte activité humaine et des enjeux écologiques majeurs. Les gestionnaires du domaine public fluvial de la Loire moyenne veillent à ce que la Loire remplisse les multiples fonctions économiques (en particulier l'assurance d'un débit minimum pour le refroidissement des centrales nucléaires), culturelles et écologiques, tout en limitant au maximum les risques d'inondation pour les populations riveraines. Ces exigences conduisent à des entretiens réguliers 
du lit de la Loire, réalisés par les Directions départementales des Territoires (DDT), pour faciliter l'écoulement de l'eau.

À proximité des agglomérations et sur les sites faciles d'accès, l'impact anthropique sur l'écosystème, et notamment sur la flore, est supposé particulièrement fort. Les sentiers, le long des berges, sont des lieux de promenade fréquentés et les espaces adjacents sont soumis au piétinement. Ces sentiers sont souvent également proches d'espaces intensément gérés (cultures, jardins, routes). On peut donc supposer que leur composition floristique porte les traces de cette situation via une présence accrue d'espèces rudérales et/ou exotiques. Les méthodes d'évaluation de la qualité de la ripisylve prennent notamment en compte la composition et la structure de la végétation, la présence d'espèces patrimoniales ou exogènes, les traces de perturbation anthropique ou l'abondance de la ressource (Aguiar et al. 2009; González del Tánago \& García de Jalón 2011). L'impact de la fréquentation, de la pollution par les déchets et la présence d'espèces envahissantes, ont ainsi par exemple été retenus par Pouvaret (2012) comme des indicateurs de l'état de conservation des forêts alluviales à bois tendre dans le Val d'Allier. À l'opposé, les îles de Loire, et tout particulièrement celles qui demeurent inaccessibles, sinon autrement qu'en embarcation, seraient a priori mieux préservées, c'est-à-dire subissant moins les perturbations anthropiques directes.

Cette étude a pour objectif de comparer la flore des berges à celle des îles, mieux préservées des impacts anthropiques. Elle se place dans le contexte des projets de recherche BioMareau (2012-2015) (Villar 2015) et BioMareau-II (2016-2019) (Villar 2020) qui s'intéressent à la dynamique de recolonisation de la biodiversité après travaux d'entretien du lit de la Loire. Dans ce cadre, des relevés floristiques sont réalisés sur les îles et les berges de Loire à Mareau-aux-Prés et Saint-Ay, au sein de la réserve naturelle nationale (RNN) de Saint-Mesmin (45). S'éloignant du thème principal des projets BioMareau, le présent article s'intéresse à la comparaison entre la berge et les îles. Une telle comparaison est motivée d'une part, par le fait qu'une grande partie de la réserve est constituée de linéaires de berges, majoritairement en ripisylves, et d'autre part, que la RNN, au contact et en aval de l'agglomération orléanaise, est particulièrement soumise à l'impact des activités humaines.

Notre hypothèse générale est que la biodiversité floristique des îles est mieux préservée que celle des berges (hypothèse globale $\mathrm{Hg}$ ).

Nous allons tenter de valider cette hypothèse en comparant la richesse et l'abondance des espèces patrimoniales, attendues plus importantes sur les îles (hypothèse élémentaire H1), à celles des espèces invasives et/ou exogènes (H2), attendues plus importantes sur les berges, ces dernières étant favorisées par les perturbations humaines (Lozon \& MacIsaac 1997) et les conséquences de l'urbanisation (Gavier-Pizarro et al. 2010), tout particulièrement dans le cas de ripisylves de faible largeur (Ives et al. 2011). Suivant la même logique, la quantité d'espèces oligotrophiles devrait être plus importante sur les îles(H3) et celle des eutrophiles plus importante sur les berges (H4). En complément, l'affectation des espèces à différents groupes suivant le type d'habitats ou le type biologique (longévité des individus) devrait apporter un éclairage fonctionnel complémentaire.

\section{MATÉRIEL ET MÉTHODES}

\section{ZONE D'ÉTUDE}

La RNN de Saint-Mesmin (département du Loiret), dont le périmètre actuel a été défini en 2006, est située à $10 \mathrm{~km}$ du centre d'Orléans et à environ $5 \mathrm{~km}$ de la partie à forte densité urbaine de l'agglomération. De forme linéaire, elle s'étend sur $9 \mathrm{~km}$ de Loire et couvre une superficie de 263 ha avec un périmètre de protection de 90 ha (Loiret Nature Environnement 2016). La géologie est constituée de surfaces en eau et d'alluvions actuelles et subactuelles, codées Fz par Gigout \& Desprez (1974), principalement sableuses ou graveleuses. Le climat est de type océanique altéré, avec des précipitations annuelles de $643 \mathrm{~mm}$ et une température moyenne de $11,3{ }^{\circ} \mathrm{C}$ (période 1981-2010 à Orléans, Météo France 2018).

Le complexe des îles de Mareau se situe dans la partie aval de la réserve, sur les communes de Mareau-aux-Prés et de Saint-Ay. Il est constitué de cinq îlots présentant divers types de végétation dont le gradient de succession va des grèves basses peu végétalisées à la forêt mature à bois dur située aux niveaux topographiques supérieurs. Aux stades intermédiaires, les peupleraies sauvages y sont dans un bon état de conservation, le site étant inclus dans l'Unité Conservatoire Mareau-Saint-Mesmin du programme national de conservation des ressources génétiques du peuplier noir, sous l'égide du Ministère de l'Agriculture (Villar \& Forestier 2017).

Au nord du complexe d'îles, la berge correspond à la digue recouverte d'espaces enherbés. Au sud, la digue étant globalement en retrait, la berge abrite une ripisylve écologiquement fonctionnelle bien que plus ou moins entrouverte et de faible largeur, comprise entre la Loire et un chemin de terre (Fig. 1).

Les inventaires floristiques utilisés pour le présent article ont porté sur trois entités (Fig. 1) correspondant à la berge sud (dénommée ci-après berge) et aux îlots B-nord et B-sud, situés à des niveaux topographiques similaires (un chenal temporaire délimite les deux îlots de superficie équivalente). La berge nord n'a pas été intégrée à l'étude car elle correspond à la digue et donne directement sur le coteau. Le Tableau 1 présente l'abondance des différents types de végétation, issus de la simplification de la typologie de Cornier (2003). Les informations sont issues de la cartographie réalisée en 2014 pour les îlots B-nord et B-sud (Villar 2015) et d'une estimation a posteriori pour la berge.

La berge ressemble à l'îlot B-nord par la représentation des types peu inondables (fruticée, peupleraie sèche et forêt à bois dur), et à l'îlot B-sud par la représentation de deux types facilement inondables (phragmitaie et phalaridaie). Par ailleurs, la berge compte beaucoup moins de surface en grèves basses que les deux îlots, à cause du talus de berge dont la base ne se découvre que modérément à l'étiage. Enfin, à quelques mètres de la rive, la berge est parcourue sur toute sa longueur par un chemin de terre fréquenté par les promeneurs, avec une intensité assez forte, compte-tenu de la proximité de l'agglomération orléanaise et de la présence du "Sentier de découverte des Isles à Mareau-aux-Prés». 


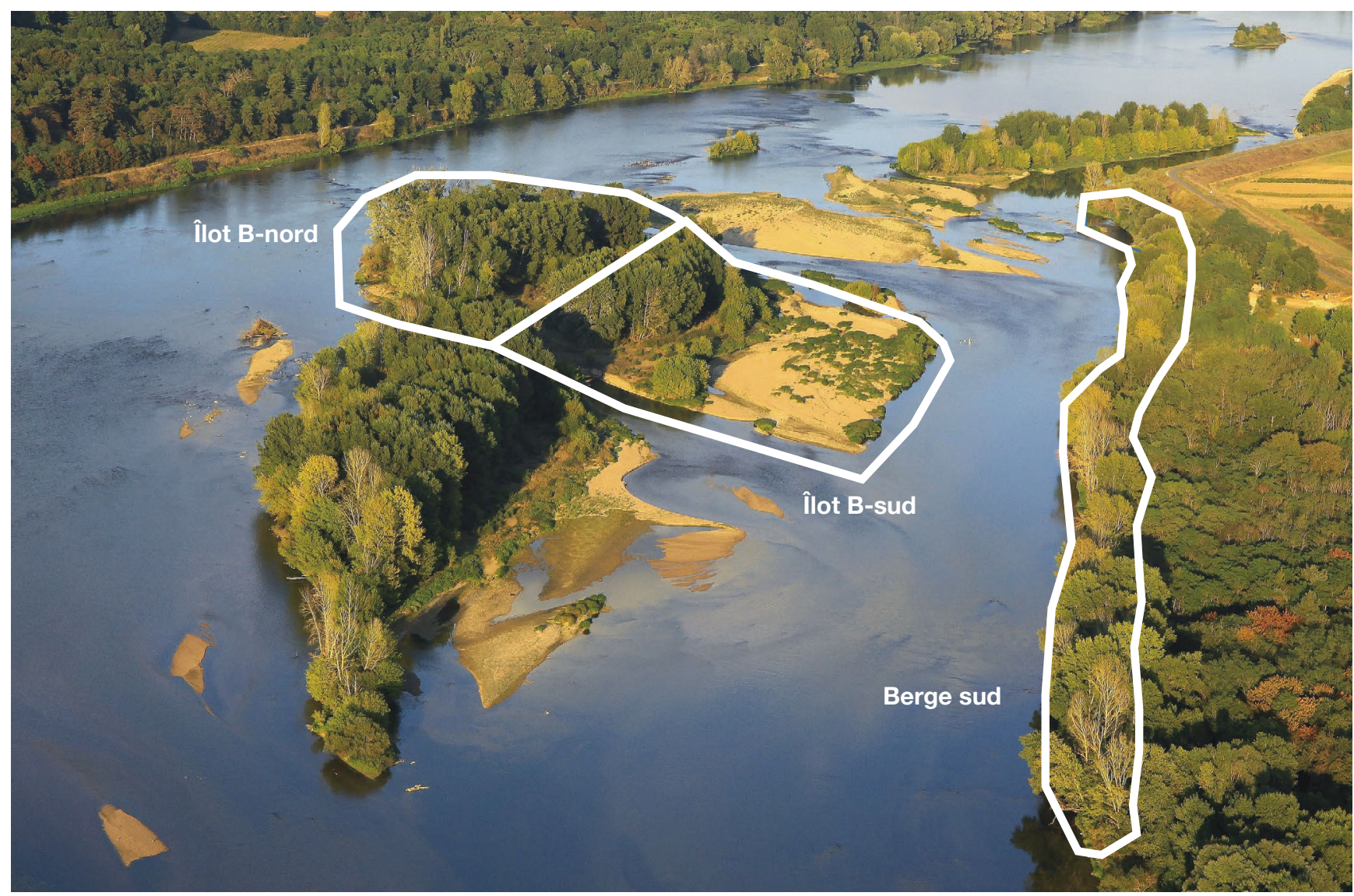

FIG. 1. - Complexe des îles de Mareau-aux-Prés en 2016 avec délimitation des entités prises en compte dans la présente étude. Crédit photo: Olivier Denux.

TABLEAU 1. - Abondance des grands types de végétation de la berge, de l'îlot B-nord et de l'îlot B-sud. Estimation de leur couvert: $\mathbf{0}$, néant; (x), inférieur à 5 \% de la surface; X, 5 à $10 \%$; XXX, 11 à $25 \%$; XXXX, supérieur à $25 \%$. Les cases grises correspondent à un couvert non accidentel (au moins $5 \%$ ).

\begin{tabular}{|c|c|c|c|c|c|}
\hline Compartiment & Types de végétation 2014 & Berge & îlot B-nord & îlot B-sud & $\begin{array}{l}\text { Habitat îlot } \\
\text { similaire à la berge }\end{array}$ \\
\hline \multirow{6}{*}{$\begin{array}{l}\text { Facilement } \\
\text { inondable }\end{array}$} & Grève basse & $(x)$ & $X X$ & $X X X$ & Néant \\
\hline & Cariçaie & $(x)$ & $(x)$ & $X X$ & (B-nord) \\
\hline & Phragmitaie & $(x)$ & 0 & $(x)$ & (B-sud) \\
\hline & Phalaridaie & $\mathrm{X}$ & $(x)$ & $\mathrm{X}$ & B-sud \\
\hline & Saulaie buissonnante & $x$ & $(x)$ & $(x)$ & Néant \\
\hline & Peupleraie du lit mineur & $\mathrm{X}$ & $X X$ & $X X$ & B-nord et B-sud \\
\hline $\begin{array}{l}\text { Moyennement } \\
\text { inondable }\end{array}$ & Prairie à chiendent & $\mathrm{X}$ & $\mathrm{X}$ & $\mathrm{X}$ & B-nord et B-sud \\
\hline Faiblement & Fruticée à rosacées & $X X$ & $X X$ & $(x)$ & B-nord \\
\hline \multirow[t]{3}{*}{ inondable } & Peupleraie «sèche» & $\mathrm{X}$ & $\mathrm{X}$ & $(x)$ & B-nord \\
\hline & Forêt à bois dur & $x X$ & $x$ & 0 & B-nord \\
\hline & Chemin pour partie enherbé & $\mathrm{X}$ & 0 & 0 & Néant \\
\hline
\end{tabular}

À l'opposé, les îlots B-nord et B-sud sont peu accessibles aux promeneurs en raison des veines d'eau à courant vif. Le caractère insulaire y favorise les grèves dont le contour peut fluctuer d'une année à l'autre (en particulier pour B-sud). Les trois entités ont subi quelques travaux d'entretien assez récents mais localisés: réouverture en 2003 d'un chenal entre les îlots B-nord et B-sud ainsi qu'à travers l'îlot B-nord et stabilisation en 2015 de la base de la digue avec des enrochements calcaires, uniquement pour l'extrémité amont de la berge (M. Chantereau comm. pers.).
RELEVÉS FLORISTIQUES

Les inventaires ont porté sur la flore vasculaire (presque exclusivement des plantes à fleurs) enracinée dans le substrat, soit terrestre, soit aquatique mais n'appartenant pas aux bras d'eau actifs au moment du relevé.

Sachant que notre préoccupation est d'inventorier le plus grand nombre d'espèces en détectant les plus rares, nous avons opté pour des relevés à l'échelle de l'assemblage des communautés ou écocomplexe (Blandin \& Lamotte 1988). Concrètement, l'unité inventoriée est l'entité îlot ou berge: 
2,05 ha pour l'îlot B-nord, 2,75 ha pour l'îlot B-sud et environ 1,5 ha pour la berge. En nous référant à Güler et al. (2016), nous considérons ici que la moindre accumulation du nombre d'espèces sur la berge, en raison de sa moindre superficie, est contre-balancée par l'effet d'accumulation lié à l'étirement en longueur, propice à l'hétérogénéité spatiale.

La reproductibilité et la représentativité de la méthode d'inventaire ont été testées et validées antérieurement (Chevalier et al. 2010b). La reproductibilité de l'observation est optimisée en effectuant trois passages annuels couvrant la phénologie de la flore ligérienne (idéalement fin juin, fin juillet et début septembre). À chaque passage et pour chaque entité, l'effort de recherche est de $1 \mathrm{~h} 45$ à deux personnes (hors détermination) ou exceptionnellement de $2 \mathrm{~h} 40$ à une personne, ce qui permet de prospecter efficacement l'ensemble de l'écocomplexe. Par ailleurs, un botaniste (R. Chevalier) a participé à tous les relevés, ce qui réduit les dérives dues aux effets opérateurs. Lors de chaque passage, un code d'abondance est attribué à chaque espèce: 1 pour accidentel, 2 pour peu fréquent, 3 pour moyennement fréquent et 4 pour fréquent.

Pour la présente étude, les relevés de 2013, 2014, 2015 et 2016 ont été utilisés. Pour chaque écocomplexe (berge, îlots B-nord et B-sud), les trois passages annuels ont été agrégés en conservant, pour chaque espèce et chaque année, le code d'abondance saisonnier le plus élevé.

\section{LES INDICES DE BIODIVERSITÉ UTILISÉS}

Pour décrire les communautés végétales rencontrées, nous avons utilisé 15 indices de richesse spécifique, tout d'abord la totalité des espèces (RS totale) puis différents groupes d'espèces à signification de patrimonialité-banalité (inspiré de Chevalier [2011] et Chevalier et al. [2010a]), d'habitat, trophique (suivant les cotations autécologiques d'Ellenberg et al. [1992]) ou biologique (issus de la simplification des types biologiques de Raunkiaer [1934], suivant les informations fournies par Julve [1998]) (Chevalier et al. 2010b).

\section{Richesse spécifique de différents groupes d'espèces à signification de patrimonialité-banalité}

RS Patri-strict. Espèces patrimoniales strictes, protégées sur l'ensemble du territoire national (Journal officiel de la République française 2013) ou en région Centre-Val de Loire (Journal officiel de la République française 1993), ou menacées, ou quasi menacées en région Centre (Cordier 2013). Exemple: Pulicaria vulgaris Gaertn. (protégée en France métropolitaine, Fig. 2).

RS Patri-élargi. Espèces patrimoniales élargies, incluant les espèces précédentes et les espèces déterminantes ZNIEFF en région Centre-Val de Loire (Cordier \& Filoche 2016). Exemple: Cyperus michelianus (L.) Link (déterminante ZNIEFF). RS Invasives. Espèces appartenant aux listes hiérarchisées d'espèces invasives (avérées ou en en observation) du CentreVal de Loire (Desmoulins \& Emeriau 2017) ou du bassin Loire-Bretagne (Varray 2017). Exemple: Acer negundo L. (invasive dans les deux listes; Fig. 3).

RS Exogènes. Espèces exogènes en région Centre-Val de Loire d'après Cordier \& Filoche (2016). Exemple: Berteroa incana (L.) DC.
Richesse spécifique de différents groupes d'espèces

à signification d'habitat

RS Cultures. Espèces des cultures, qui sont à la fois au moins assez communes dans les cultures en France d'après Jauzein (1995) et très communes en région Centre-Val de Loire d'après Cordier et al. (2010). Exemple: Tripleurospermum inodorum (L.) Sch.Bip.

RS Aqua-méga. Espèces des milieux aquatiques et des formations à grandes herbes telles que les cariçaies, les roselières, les mégaphorbiaies et les prairies longuement inondables d'après Julve (1998). Ex. : Phalaris arundinacea L.

RS Grèv-Pel. Espèces des milieux ouverts des grèves et pelouses, plus ou moins humides, à faible développement herbacé: friches annuelles, tonsures, prairies hydrophiles sur-piétinées, ou pelouses d'après Julve (1998). Exemple: Corrigiola littoralis L. RS Prair-frich. Espèces des prairies (hygrophiles ou mésophiles) et des friches vivaces (non de lisière) d'après Julve (1998). Exemple: Plantago lanceolata L. (prairie).

RS Lis-For. Groupe incluant les espèces forestières ou de lisières. Nous incluons dans les forestières, les arbres pouvant atteindre une hauteur de $16 \mathrm{~m}$, les espèces d'ombre ou de semi-ombre d'après Rameau et al. (1989) et les espèces ayant une préférence pour les forêts anciennes dans au moins trois des 22 publications examinées par Hermy et al. (1999). Pour les espèces restantes, nous incluons pour les lisières, toutes les espèces ligneuses et les espèces herbacées qui, d'après Julve (1998), ont une préférence pour les ourlets, les landes, les lisières et les clairières. Exemple: Glechoma hederacea L. (lisières).

\section{Richesse spécifique de différents groupes d'espèces à signification trophique}

RS Oligotrophiles. Espèces dont la note $\mathrm{N}$ de nutriments du sol est inférieure à 4. Exemple: Achillea ptarmica L. $(\mathrm{N}=2)$. RS Eutrophiles. Espèces dont la note $\mathrm{N}$ de nutriments du sol est supérieure à 7. Exemple: Urtica dioica L. $(\mathrm{N}=9)$.

\section{Richesse spécifique de différents groupes d'espèces à signification biologique}

RS An-Bis. Espèces annuelles ou bisannuelles. Exemple: Persicaria lapathifolia (L.) Delarbre.

RS Herb-Viv. Espèces herbacées vivaces. Exemple: Artemisia vulgaris $\mathrm{L}$.

RS Ligneuses. Espèces ligneuses. Exemple: Populus nigra L.

\section{Similitude floristique}

Toutes espèces confondues, nous avons aussi utilisé l'indice de similitude floristique de Jaccard (1901), deux à deux, entre les trois entités, en comparaison de l'effet interannuel.

\section{ANALYSE DES DONNÉES}

Une des difficultés dans l'inventaire des écocomplexes est que les entités sont en faible effectif (ici neuf relevés annuels au total), ce qui est assez peu adapté à la mise en œuvre de tests statistiques permettant de discriminer les modalités. Par ailleurs, afin de nous affranchir des variations de composition annuelle, que l'on sait relativement fortes en contexte ligérien du fait des crues et étiages plus ou moins prononcés suivant 


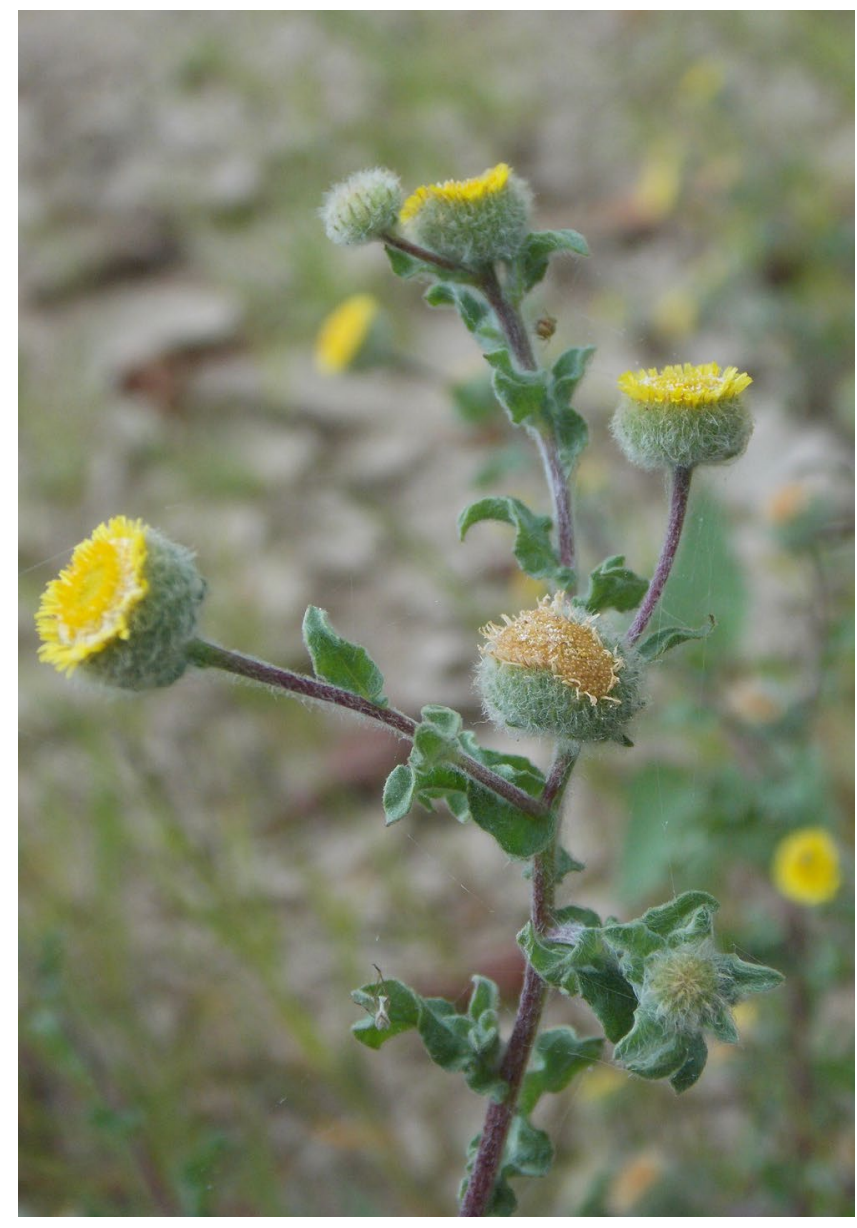

FIG. 2. - L'Herbe de Saint-Roch (Pulicaria vulgaris Gaertn.), protégée en France métropolitaine, est l'espèce patrimoniale la plus constante sur le site, rencontrée aussi bien sur les îles que sur la berge. Crédit photo: R. Chevalier.

les années (Villar 2015), nous avons fait le choix de cumuler les codes d'abondance des années 2013, 2014, 2015 et 2016. Une analyse globale des variations interannuelles est toutefois présentée pour relativiser l'importance de l'effet berge ou îlot sur la composition floristique. En conséquence, nos analyses de données seront surtout descriptives. Nous utiliserons seulement le test de probabilité exacte de Fisher lorsque nous comparerons les proportions des différents groupes d'espèces de la berge à celles des îlots. La séquence des résultats consistera à :

- examiner les valeurs de richesse atteintes par les différents groupes d'espèces pour chacune des trois entités berge, îlot B-nord et îlot B-sud;

- comparer les espèces originales (Vanpeene-Bruhier et al. 1998) entre la berge (observées uniquement sur la berge) et les deux îlots agrégés (observées sur l'un ou l'autre des deux îlots mais pas sur la berge), ce qui permet de mettre en évidence la différence de composition se produisant avec des espèces souvent peu abondantes, voire accidentelles;

- comparer le cortège des espèces préférentielles de la berge, à celui des espèces préférentielles des deux îlots agrégés. Pour cela, à partir du cumul des codes d'abondance des quatre années, pouvant atteindre 16 pour la berge et 32 pour les deux îlots agrégés (somme ensuite divisée par deux pour avoir la

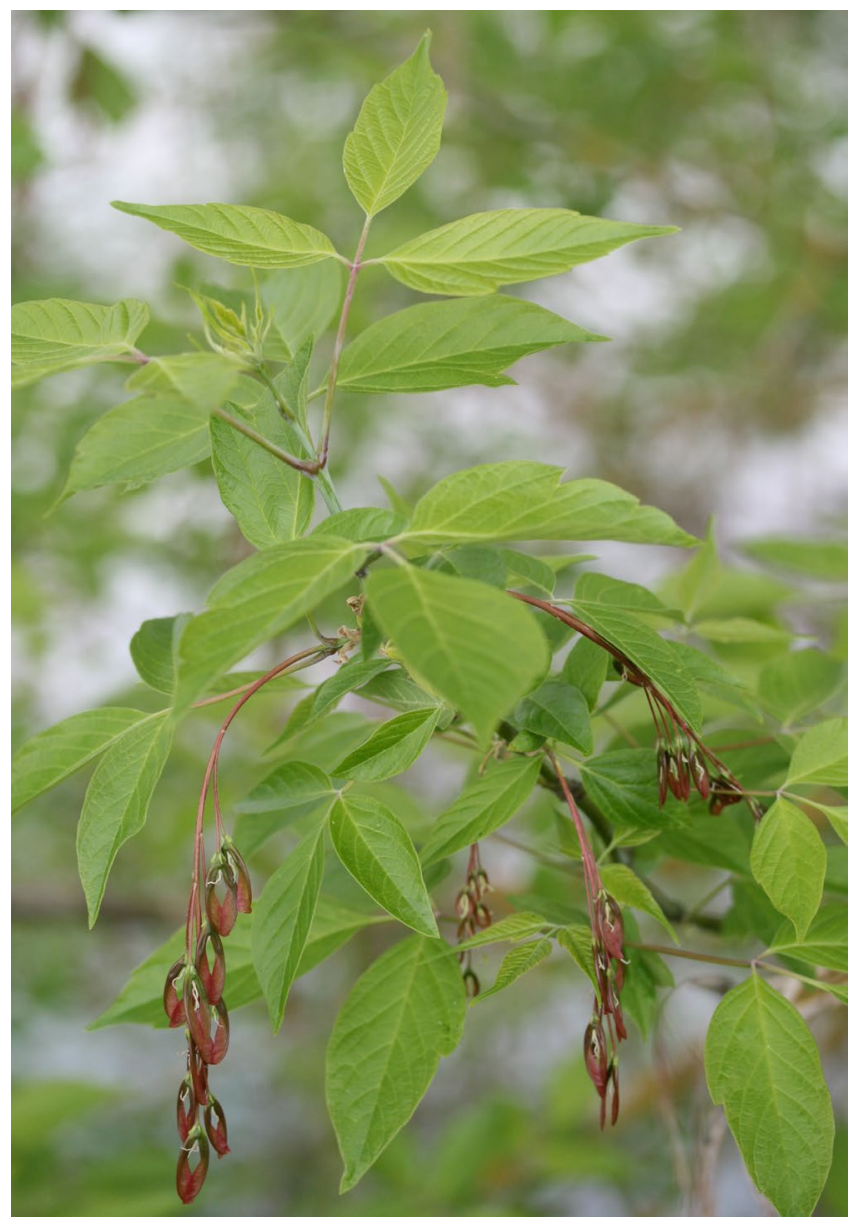

FIG. 3. - L'Érable negundo (Acer negundo L.) est l'espèce invasive la plus abondante sur le site, rencontrée aussi bien sur les îles que sur la berge. Crédit photo: R. Dupré.

même représentativité que pour la berge), nous avons considéré comme espèces préférentielles celles qui ont au moins une valeur double et un écart d'au moins quatre unités en faveur de la berge ou des îlots. Cette méthode permet, en complément de l'étude des espèces originales, de s'intéresser à des espèces mieux représentées et à caractère plus fonctionnel dans les communautés.

\section{RÉSULTATS}

\section{RÉSULTATS GÉNÉRAUX}

Pendant les quatre années 2013, 2014, 2015 et 2016, pour les trois entités berge, îlot B-nord et îlot B-sud, 418 espèces ont été inventoriées sur une superficie de 6,3 ha. 156 espèces sont originales de la berge ou de l'ensemble des deux îlots et 78 espèces sont préférentielles soit de la berge, soit des îlots. 219 espèces ne sont ni originales ni préférentielles, nous considèrerons qu'elles constituent le fond commun à la berge et aux îlots. Nous comptabilisons huit espèces patrimoniales strictes et 19 espèces patrimoniales élargies. Ces valeurs dénotent une biodiversité floristique plutôt remarquable en cohérence avec le statut de RNN. À titre de comparaison, 
TABLEAU 2. - Similitude de Jaccard, entre la berge, l'îlot B-nord et l'îlot B-sud, pour les quatre années agrégées.

\begin{tabular}{lcc}
\hline Entités & B-nord & B-sud \\
\hline Berge & 0,62 & 0,62 \\
B-nord & $/$ & 0,72 \\
\hline
\end{tabular}

nos inventaires de la berge et des deux îlots représentent les deux tiers des 630 espèces végétales vasculaires connues sur les 263 ha de la RNN (Loiret Nature Environnement 2016). Nous avons par ailleurs inventorié 62 espèces exogènes soit $15 \%$ du nombre total d'espèces, 19 d'entre-elles appartiennent aux listes d'espèces invasives en Centre-Val de Loire ou du bassin Loire-Bretagne, soit près d'un tiers des espèces figurant dans ces listes.

\section{Similitude DE JACCARD}

Nous avons calculé l'indice de Jaccard entre les trois entités berge, îlot B-nord et îlot B-sud, pour les quatre années agrégées (Tableau 2) et entre les quatre années 2013, 2014, 2015 et 2016, pour les trois entités agrégées (Tableau 3 ). Nous constatons que l'effet année est moindre (similitude comprise entre 71 et $78 \%$ ) à l'effet entité (62 à $72 \%$ ). Si la similitude de $72 \%$ entre les îlots B-nord et B-sud est incluse dans la fourchette de l'effet année, la similitude entre la berge et l'un ou l'autre des deux îlots (62\%) est inférieure. Sachant que l'abondance des types de végétation de la berge est intermédiaire entre celle des îlots B-nord et B-sud (Tableau 1), cela révèle une différence de composition entre berge et îlots.

\section{RICHESSE SPÉCIFIQUE DE LA BERGE,}

\section{DE L'ÎLOT B-NORD ET DE L'ÎLOT B-SUD}

Le Tableau 4 présente les valeurs prises par les 15 indices de richesse pour la berge, l'îlot B-nord et l'îlot B-sud. Comprise entre 308 et 321 espèces, la richesse totale varie peu, la berge atteignant une valeur intermédiaire entre les deux îlots. Les proportions de chacun des groupes d'espèces (non présentées ici) ne sont pas significativement différentes à l'exception du groupe des espèces oligotrophiles, uniquement entre la berge (8\%) et l'îlot B-nord (14\%, avec $\mathrm{p}=0,0314)$.

Cependant, la richesse des groupes d'espèces qui, pour la berge, se situe au-delà des bornes de RS entre B-sud et B-nord, nous apporte des informations tendancielles. Ainsi, pour les groupes à signification de patrimonialité-banalité, la berge compte moins d'espèces patrimoniales strictes (3 contre 4-6) et patrimoniales élargies ( 9 contre 11-14) mais aussi, moins d'espèces invasives ( 15 contre 18 ) et exogènes ( 46 contre $49-50)$ que chacun des deux îlots. Pour les groupes à signification d'habitats, la berge compte moins d'espèces des grèves-pelouses (56 contre 64-70) et plus d'espèces des cultures (67 contre 57-64), des prairies-friches ( 52 contre 47-51) et des lisières-forêts (83 contre 75-80). Pour les indices à signification trophique, la berge compte moins d'espèces oligotrophiles (26 contre 35-44) et plus d'espèces eutrophiles (63 contre 57-58). Pour les indices à signification biologique, la berge compte moins d'espèces annuelles-bisannuelles (125 contre 130-144) et plus
TABLEAU 3. - Similitude de Jaccard, entre les années 2013, 2014, 2015 et 2016, pour les trois entités agrégées.

\begin{tabular}{lccc}
\hline Années & $\mathbf{2 0 1 4}$ & $\mathbf{2 0 1 5}$ & $\mathbf{2 0 1 6}$ \\
\hline 2013 & 0,76 & 0,71 & 0,72 \\
2014 & $/$ & 0,76 & 0,74 \\
2015 & $/$ & $/$ & 0,78 \\
\hline
\end{tabular}

d'espèces herbacées-vivaces (149 contre 137-141). Si les écarts mentionnés ci-dessus ont une faible amplitude, ils tendent globalement à faire ressortir le caractère ligérien plus affirmé pour les îlots et plus généraliste pour la berge.

Les huit espèces patrimoniales strictes (Tableau 5) appartiennent majoritairement à la famille des astéracées, ont un cycle de vie court (annuelles ou bisannuelles) et sont le plus souvent inféodées aux pelouses. Seule Pulicaria vulgaris, espèce annuelle des grèves, est commune aux trois entités. Cinq espèces patrimoniales, principalement annuelles-bisannuelles des pelouses, sont rencontrées seulement dans les îlots, l'îlot B-nord étant le plus riche avec ces cinq espèces présentes. Inula britannica L. et Thalictrum flavum L., toutes deux hémicryptophytes des formations à hautes herbes, sont rencontrées uniquement sur la berge dans cette étude. Si la berge compte moins d'espèces patrimoniales strictes, elle apporte un complément de patrimonialité par rapport aux îlots.

Les 19 espèces invasives sont listées dans le Tableau 6. Cinq espèces ont un cumul d'abondance élevé ( $\geq 10 / 16)$ pour les trois entités: Acer negundo, Bidens frondosa L., Erigeron canadensis L., Symphyotrichum lanceolatum (Willd.) G.L. Nesom et Xanthium orientale L. Trois espèces atteignent cette abondance pour chacun des deux îlots: Ambrosia artemisiifolia L., Eragrostis pectinacea (Michx.) Nees et Solidago gigantea Aiton. Trois autres espèces atteignent cette abondance uniquement pour l'îlot B-sud: Lindernia dubia (L.) Pennell, Ludwigia grandiflora (Michx.) Greuter \& Burdet et Parthenocissus inserta (A. Kern.) Fritsch. Enfin, quatre espèces sont rencontrées sur les îlots mais pas sur la berge: Parthenocissus inserta, Senecio inaequidens D.C., Berberis aquifolium Pursh et Helianthus tuberosus L. Dans le Tableau 6, l'assombrissement des cases d'abondance et leur diagonalisation font ressortir que la berge est moins impactée par les invasives que les îlots alors que l'îlot B-sud, le plus soumis aux crues, est le plus impacté.

\section{ORIGINALITÉ DE LA BERGE}

PAR RAPPORT AUX ÎLOTS B-NORD ET B-SUD AGRÉGÉS

La berge compte 52 espèces originales contre seulement 34 pour l'îlot B-nord et 26 pour B-sud, ce qui confirme la différenciation de composition floristique entre berge et îlot. Ce qui nous intéresse maintenant est de comparer l'originalité de la berge ( 52 espèces) à celle des deux îlots B-nord et B-sud agrégés (104 espèces). En référence à la richesse originale de chaque entité, le Tableau 7 nous donne les proportions des différents groupes d'espèces de la berge et des deux îlots agrégés.

Malgré le nombre conséquent d'espèces originales, seuls deux indices se distinguent par une différence significative de proportion, en faveur des îlots. Ainsi, la proportion des 
TABLEAU 4. - Richesse spécifique (RS) suivant les trois entités berge, îlot B-nord et îlot B-sud. Les quatre années sont agrégées. Abréviations: An-Bis, annuelles et bisannuelles; Aqua-méga, milieux aquatiques et à grandes herbes; Grèv-Pel, grèves et pelouses; Herb-Viv, herbacées vivaces; Lis-For, des lisières et forêts; Patri-élargi, patrimoniales élargies; Patri-strict, patrimoniales strictes; Prair-frich, prairies et friches vivaces; RS, richesse en espèces. En gras, test de Fisher significatif sur les proportions; cases jaunes, valeur de la berge inférieure à celle de chacun des deux îlots; cases grises, valeur de la berge supérieure à celle de chacun des deux îlots.

\begin{tabular}{|c|c|c|c|c|}
\hline Type d'indice & RS & Berge & B-nord & B-sud \\
\hline Neutre & RS Totale & 314 & 321 & 308 \\
\hline \multirow[t]{4}{*}{ Patrimonialité-banalité } & RS Patri-strict & 3 & 6 & 4 \\
\hline & RS Patri-élargi & 9 & 14 & 11 \\
\hline & RS Invasives & 15 & 18 & 18 \\
\hline & RS Exogènes & 46 & 50 & 49 \\
\hline \multirow[t]{5}{*}{ Habitat } & RS Cultures & 67 & 64 & 57 \\
\hline & RS Aqua-méga & 47 & 38 & 49 \\
\hline & RS Grèv-Pel & 56 & 70 & 64 \\
\hline & RS Prair-frich & 52 & 51 & 47 \\
\hline & RS Lis-For & 83 & 80 & 75 \\
\hline \multirow[t]{2}{*}{ Trophique } & RS Oligotrophiles & 26 & 44 & 35 \\
\hline & RS Eutrophiles & 63 & 57 & 58 \\
\hline \multirow{3}{*}{ Biologique } & $\mathrm{RS}$ An-Bis & 125 & 144 & 130 \\
\hline & RS Herb-Viv & 149 & 137 & 141 \\
\hline & RS Ligneuses & 40 & 40 & 37 \\
\hline
\end{tabular}

TABLEAU 5. - Liste des espèces patrimoniales strictes avec leurs caractéristiques et leurs abondances. Statut patrimonial: EN, en danger; LR, liste rouge en région Centre-Val de Loire; NT, quasi menacé; Prot. N, protégé en France; Prot. RC, protégé en région Centre-Val de Loire; VU, vulnérable. Habitat: grandherb., grandes herbes. Type biologique: hémicrypto., hémicryptophyte. N Ellenberg, cotation autécologique $\mathrm{N}$ de disponibilité en nutriments du sol d'Ellenberg et al. (1992) allant de 1 (hyperoligotrophile) à 9 (polytrophile). Cases grises, cumul codes abondance, valeur comprise entre 1 et 9.

\begin{tabular}{|c|c|c|c|c|c|c|c|c|}
\hline \multirow{2}{*}{$\begin{array}{l}\text { Nom latin } \\
\text { TAXREF v11.0 }\end{array}$} & \multirow[b]{2}{*}{ Nom français } & \multirow{2}{*}{$\begin{array}{l}\text { Statut } \\
\text { patrimonial }\end{array}$} & \multirow{2}{*}{ Habitat } & \multirow[b]{2}{*}{ N Ellenberg } & \multirow[b]{2}{*}{ Type biologique } & \multicolumn{3}{|c|}{ Cumul codes abondance } \\
\hline & & & & & & Berge & B-nord & B-sud \\
\hline $\begin{array}{l}\text { Pulicaria vulgaris } \\
\text { Gaertn. }\end{array}$ & $\begin{array}{l}\text { Herbe de Saint- } \\
\text { Roch }\end{array}$ & Prot. N & grève & 7 & annuelle & 4 & 3 & 8 \\
\hline Inula britannica L. & Inule des fleuves & LR VU & grand-herb. & 5 & hémicrypto. & 2 & 0 & 0 \\
\hline Thalictrum flavum $\mathrm{L}$. & Pigamon jaune & Prot. RC & grand-herb. & 5 & hémicrypto. & 3 & 0 & 0 \\
\hline Centaurea stoebe L. & $\begin{array}{l}\text { Centaurée } \\
\text { maculée }\end{array}$ & LR EN & pelouse & / & bisannuelle & 0 & 2 & 0 \\
\hline Sedum sexangulare L. & $\begin{array}{l}\text { Orpin de } \\
\text { Bologne }\end{array}$ & LR VU & pelouse & 1 & hémicrypto. & 0 & 1 & 0 \\
\hline Crepis foetida L. & Crépide fétide & LR EN & pelouse & 3 & annuelle & 0 & 4 & 1 \\
\hline $\begin{array}{l}\text { Cynoglossum } \\
\text { officinale L. }\end{array}$ & $\begin{array}{l}\text { Cynoglosse } \\
\text { officinale }\end{array}$ & LR NT & friche & 7 & bisannuelle & 0 & 6 & 5 \\
\hline $\begin{array}{l}\text { Micropyrum tenellum } \\
\text { (L.) Link }\end{array}$ & $\begin{array}{l}\text { Catapode des } \\
\text { graviers }\end{array}$ & LR NT & pelouse & / & annuelle & 0 & 3 & 6 \\
\hline
\end{tabular}

espèces originales est très significativement supérieure pour les espèces oligotrophiles et significativement supérieure pour les espèces annuelles-bisannuelles. Ces résultats sont en accord avec ceux déjà présentés pour la richesse spécifique. La liste des espèces originales de la berge figure en Annexe 1 et celle des espèces originales des îlots en Annexe 2.

\section{ESPÈCES PRÉFÉRENTIELLES DE LA BERGE PAR RAPPORT}

À CELLES DES DEUX ÎLOTS B-NORD ET B-SUD AGRÉGÉS

78 espèces sont préférentielles soit de la berge, soit des îlots agrégés. Le Tableau 8 présente la richesse et la proportion des différents groupes d'espèces pour la berge et les îlots agrégés. Les Annexes 3 et 4 listent les espèces préférentielles.

Si la richesse totale des espèces préférentielles est proche entre berge (36) et îlots (42), nous constatons qu'un nombre conséquent d'indices (cinq) présente des différences significatives bien que les effectifs soient peu élevés. Aucun indice à signification de patrimonialité/banalité ne présente de différence significative. Aucune espèce invasive n'est préférentielle de la berge alors que quatre le sont pour les îlots: Berberis aquifolium, Eragrostis pectinacea, Lindernia dubia et Parthenocissus inserta. Les deux dernières sont préoccupantes pour le site, Lindernia dubia est massivement représentée dans la banque de graines des sédiments des grèves (Greulich et al. 2019), Parthenocissus inserta est une liane à fort développement dans les boisements alluviaux.

En parallèle, seules deux espèces patrimoniales strictes, toutes deux quasi menacées en région (NT), ont été rencontrées préférentiellement sur les îlots: Cynoglossum officinale L. dans la forêt à bois tendre et Micropyrum tenellum (L.) Link sur les pelouses sèches des grèves hautes.

Pour les indices à signification d'habitats, la proportion des espèces préférentielles est très significativement plus élevée pour les espèces des grèves-pelouses dans les îlots et significativement plus élevée pour les espèces aquatiquesmégaphorbiaies sur la berge. 
TABLEAU 6. - Liste des espèces invasives (Liste Invas.) avec leurs caractéristiques et leurs abondances. Abréviations: BLB, Bassin Loire-Bretagne; CVL, Centre-Val de Loire. Habitat: fourré-lis., fourrés et lisières; grand-herb., grandes herbes. $\mathrm{N}$ Ellenberg: cotation autécologique $\mathrm{N}$ de disponibilité en nutriments du sol d'Ellenberg et al. (1992) allant de 1 (hyperoligotrophile) à 9 (polytrophile). Type biologique: hémicrypto., hémicryptophyte. Cases grises, cumul codes abondance; gris clair, valeur comprise entre 1 et 9 ; gris foncé, valeur supérieure ou égale à 10 .

\begin{tabular}{|c|c|c|c|c|c|c|c|c|}
\hline \multirow{2}{*}{$\begin{array}{l}\text { Nom latin } \\
\quad \text { TAXREF v11.0 } \\
\end{array}$} & \multirow[b]{2}{*}{ Nom français } & \multirow[b]{2}{*}{ Liste Invas. } & \multirow[b]{2}{*}{ Habitat } & \multirow[b]{2}{*}{ N Ellenberg } & \multirow[b]{2}{*}{ Type biologique } & \multicolumn{3}{|c|}{ Cumul codes abondance } \\
\hline & & & & & & Berge & B-nord & B-sud \\
\hline Acer negundo L. & Érable negundo & CVL-BLB & forêt & 7 & ligneux & 12 & 16 & 16 \\
\hline Bidens frondosa $\mathrm{L}$. & Bident feuillé & BLB & grève & 8 & annuelle & 11 & 10 & 13 \\
\hline $\begin{array}{l}\text { Erigeron } \\
\quad \text { canadensis L. }\end{array}$ & $\begin{array}{l}\text { Conyze du } \\
\text { Canada }\end{array}$ & BLB & culture & 5 & annuelle & 11 & 12 & 11 \\
\hline $\begin{array}{l}\text { Symphyotrichum } \\
\text { lanceolatum } \\
\text { (Willd.) } \\
\text { G.L.Nesom }\end{array}$ & Aster lancéolé & CVL-BLB & grand-herb. & 8 & hémicrypto. & 15 & 12 & 13 \\
\hline $\begin{array}{l}\text { Xanthium orientale } \\
\text { L. }\end{array}$ & $\begin{array}{l}\text { Lampourde à } \\
\text { gros fruits }\end{array}$ & BLB & grève & / & annuelle & 12 & 13 & 16 \\
\hline $\begin{array}{l}\text { Ambrosia } \\
\quad \text { artemisiifolia L. }\end{array}$ & Ambroise élevée & CVL-BLB & grève & 6 & annuelle & 6 & 11 & 12 \\
\hline $\begin{array}{l}\text { Eragrostis } \\
\quad \text { pectinacea } \\
\text { (Michx.) Nees }\end{array}$ & $\begin{array}{l}\text { Éragrostis en } \\
\text { peigne }\end{array}$ & BLB & grève & / & annuelle & 4 & 13 & 15 \\
\hline $\begin{array}{l}\text { Solidago gigantea } \\
\text { Aiton }\end{array}$ & Tête d'or & CVL-BLB & grand-herb. & 7 & hémicrypto. & 6 & 10 & 12 \\
\hline $\begin{array}{l}\text { Lindernia dubia } \\
\text { (L.) Pennell }\end{array}$ & $\begin{array}{l}\text { Lindernie } \\
\text { fausse- } \\
\text { gratiole }\end{array}$ & BLB & grève & / & annuelle & 3 & 7 & 10 \\
\hline $\begin{array}{l}\text { Ludwigia } \\
\text { grandiflora } \\
\text { (Michx.) Greuter } \\
\text { \& Burdet }\end{array}$ & $\begin{array}{l}\text { Jussie à } \\
\text { grandes fleurs }\end{array}$ & CVL-BLB & grand-herb. & / & hémicrypto. & 8 & 7 & 10 \\
\hline $\begin{array}{l}\text { Parthenocissus } \\
\text { inserta (A.Kern.) } \\
\text { Fritsch }\end{array}$ & $\begin{array}{l}\text { Vigne-vierge } \\
\text { commune }\end{array}$ & CVL-BLB & / & I & ligneux & 0 & 8 & 10 \\
\hline $\begin{array}{l}\text { Elodea nuttalii } \\
\text { (Planch.) H. St. } \\
\text { John }\end{array}$ & $\begin{array}{l}\text { Élodée à feuilles } \\
\text { étroites }\end{array}$ & CVL-BLB & aquatique & 7 & hémicrypto. & 4 & 1 & 2 \\
\hline $\begin{array}{l}\text { Cuscuta } \\
\text { campestris } \\
\text { Yunck. }\end{array}$ & $\begin{array}{l}\text { Cuscute des } \\
\text { champs }\end{array}$ & BLB & / & / & annuelle & 4 & 8 & 6 \\
\hline $\begin{array}{l}\text { Erigeron } \\
\text { sumatrensis } \\
\text { Retz. }\end{array}$ & $\begin{array}{l}\text { Vergerette de } \\
\text { Barcelone }\end{array}$ & BLB & friche & / & bisannuelle & 1 & 2 & 1 \\
\hline $\begin{array}{l}\text { Reynoutria } \\
\text { japonica Houtt. }\end{array}$ & $\begin{array}{l}\text { Renouée du } \\
\text { Japon }\end{array}$ & CVL-BLB & fourré-lis. & 7 & géophyte & 1 & 2 & 1 \\
\hline $\begin{array}{l}\text { Robinia } \\
\quad \text { pseudoacacia L. }\end{array}$ & $\begin{array}{l}\text { Robinier faux- } \\
\text { acacia }\end{array}$ & CVL-BLB & forêt & 8 & ligneux & 8 & 6 & 6 \\
\hline $\begin{array}{l}\text { Senecio } \\
\text { inaequidens } \\
\text { DC. }\end{array}$ & $\begin{array}{l}\text { Séneçon sud- } \\
\text { africain }\end{array}$ & BLB & friche & 3 & hémicrypto. & 0 & 3 & 3 \\
\hline $\begin{array}{l}\text { Berberis } \\
\text { aquifolium } \\
\text { Pursh }\end{array}$ & Faux Houx & CVL & fourré-lis. & / & ligneux & 0 & 8 & 0 \\
\hline $\begin{array}{l}\text { Helianthus } \\
\quad \text { tuberosus L. }\end{array}$ & Topinambour & CVL-BLB & grand-herb. & 8 & géophyte & 0 & 0 & 1 \\
\hline
\end{tabular}

Pour les indices à signification trophique, seules les espèces eutrophiles ont une proportion significativement plus élevée pour la berge.

Pour les indices à signification biologique, la proportion des espèces annuelles-bisannuelles est hautement et significativement plus élevée pour les îlots alors que celle des herbacéesvivaces est très significativement plus élevée pour la berge.

Ces résultats confirment, complètent et élargissent ceux obtenus pour la richesse et l'originalité.
Le caractère préférentiel de la berge par rapport aux îlots est particulièrement net pour six espèces (Annexe 3, écart de cumul d'abondance $\geq 7$ ). Parmi celles-ci, Potentilla reptans L. et Lolium perenne L. sont favorisées par le chemin de terre fréquenté et tassé qui longe la berge. La berge est particulièrement propice à la diversité des saules avec cinq espèces préférentielles dont deux de façon marquée (Salix alba L. et Salix viminalis L.). La berge est aussi très favorable à Myosotis scorpioides L. qui semble apprécier son profil de transition terre/eau moyennement abrupt. 
TABLEAU 7. - Comparaison des proportions d'espèces originales (vues seulement sur la berge ou sur les deux îlots agrégés) entre la berge et les îlots agrégés pour les 15 indices de richesse. Les quatre années sont agrégées. Abréviations: An-Bis, annuelles et bisannuelles; Aqua-méga, milieux aquatiques et à grandes herbes; Grèv-Pel, grèves et pelouses; Herb-Viv, herbacées vivaces; Lis-For, des lisières et forêts; Patri-élargi, patrimoniales élargies; Patri-strict, patrimoniales strictes; Prair-frich, prairies et friches vivaces; RS, richesse en espèces. ${ }^{* *}$, test de Fisher très significatif $(p \leq 0,01)$; ${ }^{*}$, test de Fisher significatif $(p \leq 0,05)$; NS, test de Fisher non significatif $(p>0,05)$; indices et valeurs en gras, test de Fisher significatif sur les proportions; cases grises, proportions significativement supérieures.

\begin{tabular}{|c|c|c|c|c|}
\hline Signification & RS & Berge & B-nord \& B-sud & p Fisher \\
\hline Neutre & RS Originale (nombre) & 52 & 104 & / \\
\hline \multirow[t]{4}{*}{ Patrimonialité-banalité } & $\%$ RS Patri-strict & $4 \%$ & $5 \%$ & NS \\
\hline & \% RS Patri-élargi & $6 \%$ & $10 \%$ & NS \\
\hline & \% RS Invasives & $0 \%$ & $4 \%$ & NS \\
\hline & \% RS Exogènes & $13 \%$ & $15 \%$ & NS \\
\hline \multirow[t]{5}{*}{ Habitat } & \% RS Cultures & $15 \%$ & $9 \%$ & NS \\
\hline & \% RS Aqua-méga & $15 \%$ & $12 \%$ & NS \\
\hline & \% RS Grèv-Pel & $19 \%$ & $32 \%$ & NS \\
\hline & $\%$ RS Prair-frich & $19 \%$ & $15 \%$ & NS \\
\hline & \% RS Lis-For & $27 \%$ & $18 \%$ & NS \\
\hline \multirow[t]{2}{*}{ Trophique } & $\%$ RS Oligotrophiles & $10 \%$ & $30 \%$ & $\star \star$ \\
\hline & $\%$ RS Eutrophiles & $12 \%$ & $6 \%$ & NS \\
\hline \multirow[t]{3}{*}{ Biologique } & $\%$ RS An-Bis & $27 \%$ & $44 \%$ & * \\
\hline & \% RS Herb-Viv & $60 \%$ & $47 \%$ & NS \\
\hline & \% RS Ligneuses & $13 \%$ & $9 \%$ & NS \\
\hline
\end{tabular}

TABLEAU 8. - Comparaison de la richesse et des proportions d'espèces préférentielles (qui ont un écart de cumul des codes d'abondance d'au moins quatre et un rapport d'au moins deux en faveur de la berge ou des deux îlots moyennés) entre la berge et les îlots agrégés, pour les 15 indices de richesse. Les quatre années sont agrégées. Abréviations: An-Bis, annuelles et bisannuelles; Aqua-méga, milieux aquatiques et à grandes herbes; Grèv-Pel, grèves et pelouses; Herb-Viv, herbacées vivaces; Lis-For, des lisières et forêts; Patri-élargi, patrimoniales élargies; Patri-strict, patrimoniales strictes; Prair-frich, prairies et friches vivaces; RS, richesse en espèces. ${ }^{* *}$, test de Fisher hautement significatif $(p \leq 0,001)$; ${ }^{* *}$, test de Fisher très significatif $(p \leq 0,01)$; ${ }^{*}$, test de Fisher significatif $(p \leq 0,05)$; NS, test de Fisher non significatif $(p>0,05)$; indices et valeurs en gras: test de Fisher significatif sur les proportions; cases grises, proportions significativement supérieures.

\begin{tabular}{|c|c|c|c|c|c|c|}
\hline \multirow[b]{2}{*}{ Signification } & \multirow[b]{2}{*}{ RS } & \multicolumn{2}{|c|}{ RS } & \multicolumn{3}{|c|}{ \% RS Préférentielle } \\
\hline & & berge & B-nord \& B-sud & berge & B-nord \& B-sud & p Fisher \\
\hline Neutre & RS Préférentielles & 36 & 42 & $100 \%$ & $100 \%$ & / \\
\hline \multirow{4}{*}{$\begin{array}{l}\text { Patrimonialité- } \\
\text { banalité }\end{array}$} & \% RS Patri-strict & 0 & 2 & $0 \%$ & $5 \%$ & NS \\
\hline & \% RS Patri-élargi & 1 & 2 & $3 \%$ & $5 \%$ & NS \\
\hline & \% RS Invasives & 0 & 4 & $0 \%$ & $10 \%$ & NS \\
\hline & \% RS Exogènes & 4 & 8 & $8 \%$ & $19 \%$ & NS \\
\hline \multirow[t]{5}{*}{ Habitat } & \% RS Cultures & 8 & 9 & $22 \%$ & $21 \%$ & NS \\
\hline & \% RS Aqua-méga & 6 & 1 & $17 \%$ & $2 \%$ & * \\
\hline & \% RS Grèv-Pel & 2 & 15 & $6 \%$ & $36 \%$ & $\star \star *$ \\
\hline & \% RS Prair-frich & 9 & 6 & $25 \%$ & $14 \%$ & NS \\
\hline & $\%$ RS Lis-For & 11 & 10 & $31 \%$ & $24 \%$ & NS \\
\hline \multirow[t]{2}{*}{ Écologique } & \% RS Oligotrophiles & 3 & 8 & $8 \%$ & $19 \%$ & NS \\
\hline & \% RS Eutrophiles & 6 & 1 & $17 \%$ & $2 \%$ & * \\
\hline \multirow[t]{3}{*}{ Biologique } & $\%$ RS An-Bis & 8 & 28 & $22 \%$ & $67 \%$ & $\star \star *$ \\
\hline & \% RS Herb-Viv & 20 & 8 & $56 \%$ & $19 \%$ & $\star \star$ \\
\hline & \% RS Ligneuses & 8 & 6 & $22 \%$ & $14 \%$ & NS \\
\hline
\end{tabular}

Le caractère préférentiel des îlots par rapport à la berge est particulièrement net pour 15 espèces (Annexe 4), ce qui est nettement plus que pour la berge, alors que le caractère différentiel des îlots est atténué par le fait d'avoir moyenné B-nord et B-sud. Six de ces espèces sont typiques des grèves facilement inondables de Loire: Corrigiola littoralis, Plantago arenaria Waldst. \& Kit., Digitaria sanguinalis (L.) Scop., Lepidium virginicum L., Cyperus esculentus L. et Eragrostis pectinacea, les trois dernières étant naturalisées et la dernière répertoriée comme invasive mais peu préoccupante pour la RNN. Sept autres espèces à caractère préférentiel marqué sont rencontrées sur les grèves hautes à végétation plutôt xérophile, oligotrophile et à faible développement :
Chondrilla juncea L., Crepis capillaris (L.) Wallr., Echium vulgare L., Jasione montana L., Sedum acre L., Sedum album L. et Vulpia myuros (L.) C. C. Gmel.

\section{DISCUSSION}

Nos analyses ont fourni des résultats en mesure de tester les hypothèses présentées en introduction. La faible puissance statistique liée à la méthode d'inventaire et au fait d'avoir agrégé les quatre années 2013 à 2016 est compensée par la cohérence et la complémentarité des résultats obtenus suivant nos différentes approches. 
TABLEAU 9. - Synthèse du test des hypothèses formulées en introduction.

\begin{tabular}{|c|c|c|c|}
\hline Hypothèse & Intitulé & Acception & Observation \\
\hline $\mathrm{H} 1$ & $\begin{array}{l}\text { Les espèces patrimoniales sont plus abondantes } \\
\text { sur les îles }\end{array}$ & Partielle & $\begin{array}{l}\text { Tendance à plus d'espèces patrimoniales sur les } \\
\text { îles mais la berge apporte un complément non } \\
\text { négligeable }\end{array}$ \\
\hline $\mathrm{H} 2 \mathrm{a}$ & $\begin{array}{l}\text { Les espèces invasives sont plus abondantes sur } \\
\text { la berge }\end{array}$ & Contraire & $\begin{array}{l}\text { En particulier sur l'îlot B-sud en lien avec } \\
\text { l'abondance des grèves basses et les } \\
\text { perturbations hydro-sédimentaires associées }\end{array}$ \\
\hline $\mathrm{H} 2 \mathrm{~b}$ & $\begin{array}{l}\text { Les espèces exogènes sont plus abondantes sur } \\
\text { la berge }\end{array}$ & Non & Très légère tendance contraire \\
\hline $\mathrm{H} 3$ & $\begin{array}{l}\text { Les espèces oligotrophiles sont plus abondantes } \\
\text { sur les îlots }\end{array}$ & Oui & $\begin{array}{l}\text { En lien avec la plus forte abondance d'habitats de } \\
\text { grèves hautes et de pelouses }\end{array}$ \\
\hline $\mathrm{H} 4$ & $\begin{array}{l}\text { Les espèces eutrophiles sont plus abondantes } \\
\text { sur la berge }\end{array}$ & Oui & $\begin{array}{l}\text { Vraisemblablement lié à des processus } \\
\text { d'eutrophisation anthropiques locaux }\end{array}$ \\
\hline $\mathrm{Hg}$ & $\begin{array}{l}\text { La biodiversité floristique des îles est mieux } \\
\text { préservée que celle des berges }\end{array}$ & Non & $\begin{array}{l}\text { Nous ne pouvons pas conclure à un meilleur état } \\
\text { de conservation de la flore des îles par rapport } \\
\text { à la berge mais à une complémentarité de } \\
\text { composition }\end{array}$ \\
\hline
\end{tabular}

\section{PERTINENCE DU CONCEPT D'ESPÈCES PRÉFÉRENTIELLES}

Le concept d'espèces préférentielles, qui met en avant le contraste d'abondance de chaque espèce, est celui qui permet la plus forte discrimination entre berge et îlots. En toute logique, il révèle mieux le fonctionnement de l'écosystème que l'originalité du cortège floristique, dont une forte proportion est constituée d'espèces rares, opportunistes voire accidentelles.

\section{UNE DIFFÉRENCE DE COMPOSITION MODÉRÉE}

ENTRE BERGE ET ÎLE

La berge et chacun les deux îlots comptent autant d'espèces (environ 310 espèces). Cette richesse est conséquente, ce qui est habituel en contexte alluvial (Nilsson \& Svedmark 2002; Nobis et al. 2016). Sachant cela, la similitude de Jaccard entre la berge et chacun des deux îlots est plus faible que celle entre les deux îlots, alors que la berge a une représentation des habitats intermédiaire (Tableau 1). Ceci révèle une différence de composition principalement restituée par l'étude des espèces préférentielles (11\% de la richesse totale soit de la berge, soit des îlots), nous estimons cette différence comme modérée. Nous pouvons attribuer cette faible différence à la gestion très extensive de la berge, depuis une cinquantaine d'années, favorisant la dynamique forestière naturelle (D. Hémeray comm. pers.). En conséquence, nos hypothèses formulées en introduction ne sont que très partiellement validées (Tableau 9).

\section{DES SIGNES D'EUTROPHISATION SUR LA BERGE}

La principale validation concerne la plus forte abondance des espèces oligotrophiles sur les îlots et des espèces eutrophiles sur la berge. Cette apparente complémentarité pourrait avoir deux origines, tout d'abord la plus forte abondance de certains habitats sur les îlots, tels les grèves hautes et les pelouses (Tableau 1), les plus propices à une flore oligotrophile au sein de l'écocomplexe, et ensuite une eutrophisation de la berge qui nous semble surtout attribuable à des perturbations anthropiques locales et en particulier la fréquentation humaine, car moins marquée sur les îlots pourtant plus soumis aux crues véhiculant des eaux eutrophisées.

\section{UNE PLUS FORTE OCCURRENCE DES ESPÈCES INVASIVES} SUR LES ÎLES

Cependant, cette eutrophisation ne se traduit pas par une plus forte présence des espèces exogènes sur la berge, nous observons même le contraire pour les espèces invasives, davantage représentées sur les îlots. D’après Davis et al. (2000) et Shea \& Chesson (2002), la fréquence élevée des perturbations, ici d'ordre hydrologique, serait en mesure de créer des "opportunités de niche" propices à l'expression des espèces invasives. Ceci semble particulièrement vrai pour les habitats pionniers des grèves, bien plus abondants sur les îlots que sur la berge, favorables à l'installation de nouvelles espèces exogènes (Cornier 2002; Greulich et al. 2016). Ce concept serait ici illustré par le fait que l'îlot B-sud est à la fois le plus impacté par les crues et par les espèces invasives (Tableau 6). Dans leur méta-analyse, qui s'intéresse à la diversité des espèces indigènes et exotiques dans les forêts riveraines européennes à caractère naturel, Schnitzler et al. (2007) mettent en évidence que les saulaiespeupleraies, à l'aval des vallées situées aux latitudes inférieures (ce qui correspond aux conditions de notre étude), sont les plus exposées aux espèces exogènes et par conséquence aux espèces invasives. Par ailleurs, le corridor ligérien est connu pour être très favorable à la propagation des espèces invasives (Vahrameev et al. 2015; Desmoulins \& Emeriau 2017).

\section{PRÉDOMINANCE DES FACTEURS GLOBAUX}

SUR LES FACTEURS LOCAUX DE PERTURBATION

Tout cela tendrait à privilégier l'hypothèse que les conditions d'expression des espèces exogènes et invasives s'exerceraient à une échelle plus vaste et globale (Davies et al. 2005) qu'à celle d'une pression anthropique locale, ici liée à la forte fréquentation de la berge en comparaison des îlots. À cette dernière échelle, les décharges sauvages de déchets verts, pourvoyeuses de plantes exogènes et invasives (Rusterholz\& Baur 2013), sont rares et le cas échéant rapidement évacuées, en raison de la vigilance des personnels de la RNN (D. Hémeray comm. pers.) en concertation avec les différents acteurs, suivant une approche cohérente avec les recom- 
mandations de Robinson et al. (2017). De même, l'apport d'espèces d'habitats adjacents est limité sur le site d'étude par le fait que la berge est séparée des cultures par une levée qui représente une barrière physique susceptible de limiter la dispersion des graines. Dans un contexte moins surveillé, l'effet local pourrait être plus important. Ces résultats nous interpellent toutefois sur l'impact des espèces invasives sur des îlots pourtant soustraits de l'influence directe de l'homme et soumis à un régime de perturbation plutôt naturel en comparaison de celui de la berge. La problématique des espèces invasives en Loire moyenne serait donc à raisonner à une échelle étendue sur le linéaire, et cela d'autant plus que des crues morphogènes et une dynamique sédimentaire active créent des habitats pionniers de type grève, caractérisés par une végétation éparse (Cornier 2002; Greulich et al. 2016) favorables à l'implantation d'espèces rudérales et exotiques (Burkart 2001; Tabacchi et al. 2005; Rood et al. 2010).

L'effet du régime de perturbation (ici crues et étiages) serait prépondérant par rapport à celui plus local lié à la fréquentation et à l'urbanisation. Il a en effet été démontré que la dispersion des espèces par les crues le long du fleuve est un facteur important de la biodiversité (Andersson et al. 2000; Merritt \& Wohl 2002; Goodson et al. 2002, 2003; Gurnell et al. 2008; Hyslop \& Trowsdale 2012), même dans les paysages urbains fragmentés (Brice et al. 2016).

\section{LA DIFFÉRENCE ENTRE BERGE ET ÎLE REPOSE AUSSI} SUR L'ASSEMBLAGE DES HABITATS

L'étude des indices à signification d'habitats rend compte d'éléments déjà évoqués et liés à la topographie différenciée de la berge par rapport à celle des îlots : meilleure représentation des espèces des grèves et pelouses sur les îlots et, de façon moins nette, des espèces aquatiques et des hautes herbes sur les berges bénéficiant d'une topographie stable par rapport aux îlots.

Les résultats obtenus avec les indices à signification biologique sont concordants avec les précédents: meilleure représentation des espèces annuelles et bisannuelles sur les îlots et des espèces herbacées vivaces sur les berges.

LA BERGE CONTRIBUE À L'APPORT D'ESPÈCES PATRIMONIALES Concernant les espèces patrimoniales, les îlots auraient tendance à en présenter un plus grand nombre mais, compte tenu de la faible abondance de ces espèces, le principal constat est ici que les berges apportent une part non négligeable d'espèces patrimoniales en complément de celles des îlots.

\section{UN ASSEZ BON ÉTAT DE LA BERGE ET UNE CONTRIBUTION ÉCOLOGIQUE COMPLÉMENTAIRE À CELLE DES ÎLES}

Nous avions initialement un a priori sur le meilleur état de conservation de la flore des îlots par rapport à celui de la berge. Au vu de nos résultats, nous ne pouvons pas conclure à un meilleur état de l'un par rapport à l'autre. Outre le fait, qu'aussi bien les îlots que la berge présentent une biodiversité floristique intéressante en rapport avec le statut de RNN, chaque entité présente des signes de dégradation écologique (abondance des invasives sur les îlots et signes d'eutrophisation sur la berge).
Nos résultats nous conduisent à considérer la ripisylve de berge comme un milieu complémentaire des îlots boisés, y compris par son apport d'espèces patrimoniales (ici Inula britannica et Thalictrum flavum) mais aussi par sa remarquable diversité en espèces de saules, éléments structurants des cortèges dendrologiques ligériens. Au final, nous estimons que les cortèges floristiques de la berge étudiée sont plutôt en bon état compte tenu du contexte péri-urbain.

\section{DES INVESTIGATIONS COMPLÉMENTAIRES A MENER POUR GÉNÉRALISER LES RÉSULTATS}

Les résultats obtenus sont encourageants, en cohérence avec la bibliographie. Même s'ils ont été obtenus à partir d'un inventaire complet sur 6,3 hectares, ils ne sont pas généralisables à partir d'un seul cas. Précisons tout d'abord que notre étude a porté sur la comparaison de deux îlots inondables et d'une berge moyennement abrupte constituée en ripisylve, elle-même en partie inondable. Nous pensons que les entités étudiées sont assez représentatives d'un grand nombre d'îlots et de berges en ripisylve de Loire moyenne. C'est ce que nous avons pu constater lors d'un échantillonnage visant à étudier la dynamique des saulaies-peupleraies sur l'ensemble de la Loire moyenne, dans le cadre d'une autre action du projet BioMareau-II. En revanche, le contexte péri-urbain est assez singulier bien qu'il se répète tout au long de la Loire. Le contraste entre berge et îlot est-il plus fort en contexte péri-urbain que loin des agglomérations ou bien le contexte péri-urbain généralise-t-il certains processus, tels que l'eutrophisation ou l'invasibilité des habitats? Pour répondre à ces questions et vérifier si les réponses ont une portée générale, nous préconisons de réaliser des études complémentaires, en différents sites de Loire moyenne, aussi bien en contexte péri-urbain que soustrait de cette influence. Un tel dispositif, suivi annuellement, pourrait aussi contribuer à la surveillance des espèces patrimoniales et invasives en Loire moyenne, notre protocole de caractérisation se prêtant bien à la détection des espèces rares ou émergentes.

\section{CONCLUSION}

La richesse spécifique de la ripisylve de berge est assez similaire à celle des îlots étudiés, pourtant mieux préservés de la fréquentation du public. Nous constatons cependant une légère différence de composition. Celle-ci suggère un processus de banalisation des communautés végétales sur la berge, vraisemblablement lié à un phénomène d'eutrophisation, et sur les îlots, par les espèces invasives.

Toutefois, le contraste entre ripisylve de berge et îlot est moins fort que nous le supposions. Si les espèces patrimoniales ont tendance à être mieux représentées sur les îles, la berge y contribue aussi de façon complémentaire. Plus étonnant, les espèces exogènes ne sont pas davantage présentes sur la berge alors que les espèces invasives sont plus fréquentes sur les îlots.

En conséquence, la flore de la berge n'est pas dans un état aussi dégradé que nous l'imaginions et présente même des atouts complémentaires à ceux des îlots. À l'opposé, la plus 
forte abondance des espèces invasives sur les îlots nous alerte, notamment en lien avec le réchauffement climatique qui pourrait encore accentuer le processus, particulièrement pour les espèces originaires de zones climatiques plus chaudes (Dukes \& Mooney 1999). Si nos résultats ne sont pas généralisables, ils demeurent utiles pour alerter le gestionnaire sur l'intérêt des ripisylves de berge au sein de la RNN de Saint-Mesmin et l'inciter à y porter une plus grande attention. Concernant la flore vasculaire, nous pouvons formuler les constats et recommandations suivants :

- les ripisylves de berge constituent des écocomplexes linéaires intéressants à forte hétérogénéité, facteur de biodiversité;

- de ce fait, elles méritent d'être identifiées en tant qu'écocomplexes devant être cartographiés à l'aide d'une typologie préalablement établie;

- elles doivent bénéficier d'une gestion attentive, d'autant plus qu' elles sont soumises à une forte fréquentation humaine;

- le maintien et l'amélioration de leur état passe inévitablement par leur surveillance et la sensibilisation du public sur leur enjeu.

Nos résultats ne concernant que la flore vasculaire, il serait intéressant de mener des investigations pour savoir s'ils s'appliquent aussi à d'autres groupes taxonomiques, particulièrement faunistiques, afin d'avoir une vision globale du fonctionnement de l'écosystème et de formuler des préconisations de gestion consensuelles.

\section{Remerciements}

Nous remercions en premier lieu les deux rapporteurs Serge Muller et Rémy Poncet pour leurs remarques constructives et pour leur contribution à l'amélioration de la rédaction.

Nous tenons aussi à remercier les personnes qui, en plus d'une partie des auteurs (Richard Chevalier, Michel Chantereau, Rémi Dupré, Damien Hémeray et Hilaire Martin), ont apporté leur soutien lors de la réalisation des inventaires: Ophélie Beslin, Aurélie Bouvet, Adélie Chevalier, Florient Desmoulins, Félix Langlois, Pierre Payan, Coryse Pernot, Emmanuel Pineau, Sara Robert et Anne Villemey.

Cette étude rentre dans le cadre des projets de recherche BioMareau et BioMareau-II, opérations cofinancées par la Région Centre-Val de Loire et l'Union européenne, l'Europe s'engageant sur le bassin de la Loire avec le Fonds européen de développement régional.

\section{RÉFÉRENCES}

Aguiar F. C., Ferreira M. T., Albuquerque A., RodríguezGONZÁlEZ P. \& SEGURADO P. 2009. - Structural and functional responses of riparian vegetation to human disturbance: performance and spatial scale-dependence. Fundamental and Applied Limnology / Archiv für Hydrobiologie 175 (3): 249-267. https:// doi.org/10.1127/1863-9135/2009/0175-0249

Andersson E., Nilsson C. \& Johansson M. E. 2000. — Plant dispersal in boreal rivers and its relation to the diversity of riparian flora. Journal of Biogeography 27: 1095-1106. https://doi. org/10.1046/j.1365-2699.2000.00481.x

Blandin P. \& LAMOTTE M. 1988. - Recherche d'une entité écologique correspondant à l'étude des paysages: la notion d'écocomplexe. Bulletin d'Écologie 19 (4): 547-555.

Boudin L., Cordier J. \& MoreT J. 2007. - Atlas de la flore remarquable du Val de Loire entre le bec d'Allier et le bec de Vienne. Muséum national d'Histoire naturelle, Paris, 464 p.

Brice M. H., Pellerin S. \& Poulin M. 2016. - Environmental filtering and spatial processes in urban riparian forests. Journal of Vegetation Science 27: 1023-1035. https://doi.org/10.1111/ JVS. 12425

BURKART M. 2001. - River corridor plants (Stromtalpflanzen) in Central European Lowland: a review of a poorly understood plant distribution pattern. Global Ecology \& Biogeography 10 (5): 449-468. https://doi.org/10.1046/j.1466-822x.2001.00270.x

Chevalier R. 2011. - Essai de mise au point d'indices synthétiques et universels de valeur patrimoniale et de banalisation de la flore. Exemples d'application dans le département du Loiret. Symbioses, nouvelle série 27: 11-16.

Chevalier R., Berthelot A. \& Gaudin S. 2010a. - Biodiversité floristique des peupleraies dans les vallées de Champagne: comparaison avec les prairies et les forêts suivant une approche de la patrimonialité et de la banalité. Bulletin de la Société d'Étude des Sciences naturelles de Reims 24: 19-34.

Chevalier R., Gautier G. \& Archaux F. 2010b. - Relevés floristiques pour le suivi de la biodiversité végétale des écosystèmes forestiers: éléments de réflexion pour faire les bons choix. Revue forestière française LXII (2): 141-154. https://doi. org/10.4267/2042/34651

Claessens H., Rondeux J., Debruxelles N., Burton C. \& Lejeune P. 2009. - Le suivi des bandes riveraines des cours d'eau de Wallonie. Revue forestière française LXI (6): 595-610. https://doi.org/10.4267/2042/32931

CORDIER J. (COORD.) 2013. — Liste rouge des plantes vasculaires de la région Centre, in Nature Centre \& CONSERvatoire BOTANIQUE NATIONAL DU BASSIN PARISIEN (éds), Livre rouge des habitats naturels et des espèces menacées de la région Centre. Nature Centre, Orléans: 97-171.

Cordier J. \& Filoche S. 2016. - Notice du catalogue de la flore vasculaire du Centre-Val de Loire. Version mai 2016. CBNBP, Paris, $21 \mathrm{p}$.

Cordier J., Dupré R. \& Vahrameev P. 2010. — Catalogue de la flore sauvage de la région Centre. Symbioses, nouvelle série 26: 36-84.

CORNIER T. 2002. - La végétation alluviale de la Loire entre le Charolais et l'Anjou: essai de modélisation de l'hydrosystème. Thèse Université François Rabelais, Tours, 227 p.

CORNiER T. 2003. - Typologie et synthèse synsystématique de la végétation alluviale de la Loire entre le Charolais et l'Anjou. Version 2003.3. Université François Rabelais, Tours, 91 p.

Davis M. A., Grime J. P. \& ThOMPSON K. 2000. — Fluctuating resources in plant communities: a general theory of invasibility. Journal of Ecology 88: 528-534. https://doi.org/10.1046/j.13652745.2000.00473.x

Davies K. F., Chesson P., Harrison S., InOuye B. D., MelBOURNE B. A. \& RiCE K. J. 2005. — Spatial heterogeneity explains the scale dependence of the native-exotic diversity relationship. Ecology 86 (6): 1602-1610. https://doi.org/10.1890/04-1196

Desmoulins F. \& EMERIAU T. 2017. - Liste hiérarchisée des espèces végétales invasives du Centre-Val de Loire, version 3.0, novembre 2017. CBNBP, Orléans, 47p.

DiRECTION GÉNÉRALE DE L'AMÉNAGEMENT, DU LOGEMENT ET DE LA NATURE 2016. - Carte des sites Natura 2000. MNHN/INPN, Paris, données au 30 septembre 2016.

DUKES J. S. \& MOONEY H. A. 1999. - Does global change increase the success of biological invaders? Trends in Ecology \& Evolution 14 (4): 135-139. https://doi.org/10.1016/S0169-5347(98)01554-7

Ellenberg H., Weber H. E., DÜll R., Wirth V., Werner W. \& Paulissen D. 1992. - Zeigerwerte von Pflanzen in Mitteleuropa. Scripta geobotanica XVIII: 1-258.

Gavier-Pizarro G. I., Radeloff V. C., Stewart S. I., HuebNer C. D. \& Keuler N. S. 2010. - Housing is positively 
associated with invasive exotic plant species richness in New England, USA. Ecological Applications 20 (7): 1913-1925. https:// doi.org/10.1890/09-2168.1

Gigout M. \& Desprez N. 1974. - Carte géologique au 1/50 000 de Beaugency XXI-20. BRGM, Orléans, notice $15 \mathrm{p}$.

González del TÁnago M. \& García de Jalón D. 2011. Riparian Quality Index (RQI): A methodology for characterising and assessing the environmental conditions of riparian zones. Limnetica 30 (2): 235-254. https://doi.org/10.23818/limn.30.18

Goodson J. M., Gurnell A. M., Angold P. G. \& Morrissey I. P. 2002. - Riparian seed banks along the lower River Dove, UK: their structure and ecological implications. Geomorphology 47: 45-60. https://doi.org/10.1016/S0169-555X(02)00140-X

Goodson J. M., Gurnell A. M., ANGold P. G. \& Morrissey I. P. 2003. - Evidence for hydrochory and the deposition of viable seeds within winter flow-deposited sediments: the River Dove, Derbyshire, UK. River Research and Applications 19: 317-334. https://doi.org/10.1002/rra.707

Greulich S., Guitton H. \& Lacroix P. 2016. - Habitats et végétation de la plaine inondable, in MOATAR F. \& DUPONT N. (coord.), La Loire fluviale et estuarienne. Un milieu en évolution. Quae, Versailles: 174-185.

Greulich S., Chevalier R. \& Villar M. 2019. - Soil seed banks in the floodplain of a large river: A test of hypotheses on seed bank composition in relation to flooding and established vegetation. Journal of Vegetation Science 30 (4): 732-745. https:// doi.org/10.1111/jvs.12762

GÜler B., Jentsch A., Apostolova I., Bartha S., Bloor J. M. G., Campetella G., Canullo R., Házi J., Kreyling J., Pottier J., Szabó G., Terziyska T., UĞurlu E., Wellstein C., ZimmerMANN Z. \& DENGLER J. 2016. - How plot shape and spatial arrangement affect plant species richness counts: implications for sampling design and rarefaction analyses. Journal of Vegetation Science 27: 692-703. https://doi.org/10.1111/jvs.12411

Gurnell A., Thompson K., Goodson J. \& Moggridge H. 2008. - Propagule deposition along river margins: linking hydrology and ecology. Journal of Ecology 96 (3): 553-565. https:// doi.org/10.1111/j.1365-2745.2008.01358.x

Hermy M., Honnay O., Firbank L., Grashof-Bokdam C. \& LAWESSON J. E. 1999. - An ecological comparison between ancient and other forest plant species of Europe, and the implications for forest conservation. Biological Conservation 91 (1): 9-22. https://doi.org/10.1016/S0006-3207(99)00045-2

Hyslop J. \& TROWSDALE S. 2012. - A review of hydrochory (seed dispersal by water) with implications for riparian rehabilitation. Journal of Hydrology (New Zealand) 51 (2): 137-152.

Ives C. D., Hose G. C., Nipperess D. A. \& TAylor M. P. 2011. The influence of riparian corridor width on ant and plant assemblages in northern Sydney, Australia. Urban Ecosystems 14: 1-16. https://doi.org/10.1007/s11252-010-0141-8

JACCARD P. 1901. - Distribution de la flore alpine dans le bassin des Dranses et dans quelques régions voisines. Bulletin de la Société vaudoise des Sciences naturelles 37: 241-272.

JAUZEIN P. 1995. - Flore des champs cultivés. INRA, SOPRA, Paris, $898 \mathrm{p}$.

JouRnAL OFFICIEL DE LA RéPUbLIQUE FRANÇAISE 1993. — Arrêté du 12 mai 1993 relatif à la liste des espèces végétales protégées en région Centre complétant la liste nationale. Journal officiel de la République française 161: 9965.

JOURNAL OFFICIEL DE LA RéPUBLIQUe FRANÇAISE 2013. — Arrêté du 23 mai 2013 portant modification de l'arrêté du 20 janvier 1982 relatif à la liste des espèces végétales protégées sur l'ensemble du territoire national. Journal officiel de la République française 130: 9491.

JULVE P. 1998 ff. - Baseflor. Index botanique, écologique et chorologique de la flore de France. Version 07 septembre 2006. http://philippe. julve.pagesperso-orange.fr/catminat.htm

LOIRET NATURE ENVIRONNEMENT 2016. — Plan de gestion 2016-
2020 Réserve naturelle nationale de Saint-Mesmin. Loiret Nature Environnement, Orléans, $126 \mathrm{p}$.

LOZON J. D. \& MACLSAAC H. J. 1997. — Biological invasions: Are they dependent on disturbance? Environmental Reviews 5 (2): 131-144. https://doi.org/10.1139/a97-007

Merritt D. M. \& Wohl E. E. 2002. — Processes governing hydrochory along rivers: Hydraulics, hydrology, and dispersal phenology. Ecological Applications 12 (4): 1071-1087. https://doi. org/10.1890/1051-0761(2002)012[1071:PGHARH]2.0.CO;2

MéTÉO France 2018. - Météo et climat, données climatiques de la station d'Orléans. Météo-France, dernière consultation le 5 mars 2018

NAiman R. J. \& DÉCAMPS H. 1997. - The ecology of interfaces: riparian zones. Annual Review of Ecology and Systematics 28: 621658. https://doi.org/10.1146/annurev.ecolsys.28.1.621

Nilsson C. \& Svedmark M. 2002. - Basic principles and ecological consequences of changing water regimes: Riparian plant communities. Environmental Management 30 (4): 468-480. https://doi.org/10.1007/s00267-002-2735-2

Nobis A., ŻMiHORSKI M. \& KotowsKA D. 2016. — Linking the diversity of native flora to land cover heterogeneity and plant invasions in a river valley. Biological Conservation 203: 17-24. http://dx.doi.org/10.1016/j.biocon.2016.08.032

Pouvaret S. 2012. - Évaluation de l'état de conservation des forêts alluviales à bois tendre sur le site Natura 2000 Val d'Allier, de Jumeaux à Pont-du-Château. Actes des Rencontres végétales $d u$ Massif central, 28-30 avril 2010 57-61.

Rameau J. C., Mansion D., Dumé G., Timbal J., Lecointe A., DupONT R. \& KELLER R. 1989. - Flore forestière française. Guide écologique illustré. Tome 1. Plaines et collines. Institut pour le Développement Forestier, Paris, 1785 p.

RAUNKIAER C. 1934. - The Life Forms of Plants and Statistical Plant Geography. Clarendon Press, Oxford, $632 \mathrm{p}$

Robinson B. S., INGER R., CROWLEY S. L. \& GASTON K. J. 2017. Weeds on the web: conflicting management advice about an invasive non-native plant. Journal of Applied Ecology 54: 178-187. https://doi.org/10.1111/1365-2664.12712

Rood S. B., BraAtne J. H. \& Goater L. A. 2010. — Favorable fragmentation: river reservoirs can impede downstream expansion of riparian weeds. Ecological Applications 20 (6): 1664-1677. https://doi.org/10.1890/09-0063.1

Rusterholz H. P. \& Baur B. 2013. - Néophytes en forêt. Des intruses profitent des dépôts illégaux. La Forêt 65 (5): 7-9.

Sabo J. L., Sponseller R., Dixon M., Gade K., Harms T., HefFERNAN J., JaNi A., KatZ G., SOYKan C., WATTS J. \& WeLter J. 2005. - Riparian zones increase regional species richness by harboring different, not more, species. Ecology 86 (1): 56-62. https://doi.org/10.1890/04-0668

Schnitzler A., Hale B. W. \& Alsum E. M. 2007. — Examining native and exotic species diversity in European riparian forests. Biological Conservation 138: 146-156. https://doi.org/10.1016/j. biocon.2007.04.010

SHEA K. \& CHESSON P. 2002. - Community ecology theory as a framework for biological invasions. Trends in Ecology \& Evolution 17 (4): 170-176. https://doi.org/10.1016/S0169-5347(02)02495-3

Tabacchi E., Planty-Tabacchi A. M., Roques L. \& Nadal E. 2005. - Seed inputs in riparian zones: implications for plant invasion. River Research and Applications 21 (2-3): 299-313. https://doi.org/10.1002/rra.848

Vahrameev P., Nobilliaux S. \& Desmoulins F. 2015. - Liste des espèces végétales invasives de la région Centre-Val de Loire, version 2.4. Conservatoire botanique national du Bassin parisien, délégation Centre, Orléans, $41 \mathrm{p}$.

VAnpeene-Bruhier S., Moyne M. L. \& Brun J. J. 1998. La richesse spécifique: un outil pour la prise en compte de la biodiversité dans la gestion de l'espace. Application en Haute Maurienne (Aussois, Savoie). Ingénieries - E A T 15: 47-59.

VARRAY S. (COORD.) 2017. — Liste catégorisée des espèces exotiques 
envahissantes du bassin Loire-Bretagne. Version avril 2017. Fédération des Conservatoires d'espaces naturels, Orléans, $18 \mathrm{p}$.

VILLAR M. (COORD.) 2015. - BioMareau: conséquences des travaux d'entretien du lit de la Loire sur plusieurs composantes de la biodiversité au sein de la mosä̈que des îles de Mareau-aux-prés (Loiret). Compte-rendu scientifique final (juillet 2012 - juin 2015). INRA, Ardon, 194 p.

VILLAR M. (COORD.) 2020. — BioMareau-II: Dynamique de recolo- nisation de la biodiversité après travaux d'entretien du lit de la Loire. Compte-rendu scientifique final (juillet 2016 - décembre 2019). INRAE, Ardon, 223 p.

Villar M. \& ForESTIER O. 2017. — La France à la sauvegarde du Peuplier noir : état actuel du programme de conservation et de valorisation des ressources génétiques. Revue forestière française LXIX (3): 195-204. https://doi.org/10.4267/2042/65335

Soumis le 17 février 2020 accepté le 21 août 2020; publié le 6 janvier 2021. 
ANNEXES

ANNEXE 1. - Liste et caractéristiques des espèces originales de la berge par rapport aux îlots. Patrimonialité/banalité: LR, liste rouge en région Centre-Val de Loire; Prot. RC, protégé en région Centre-Val de Loire; VU, vulnérable; ZNIEFF, espèce déterminante ZNIEFF. Habitat: fourré-lis., fourrés et lisières; grandherb., grandes herbes. Type biologique: hémicrypto., hémicryptophyte.

\begin{tabular}{|c|c|c|c|c|c|}
\hline $\begin{array}{l}\text { Nom latin } \\
\text { TAXREF v11.0 }\end{array}$ & Nom français & $\begin{array}{l}\text { Patrimonialité } \\
\text { /banalité }\end{array}$ & Habitat & $\begin{array}{l}\text { Type } \\
\text { biologique }\end{array}$ & $\begin{array}{r}\text { Cumul codes } \\
\text { abondance }\end{array}$ \\
\hline Agrostis gigantea Roth & Agrostide géant & / & prairie & hémicrypto. & 1 \\
\hline Allium vineale $\mathrm{L}$. & Ail des vignes & / & culture & géophyte & 5 \\
\hline Alopecurus pratensis L. & Vulpin des prés & / & prairie & hémicrypto. & 1 \\
\hline Althaea officinalis L. & Guimauve officinale & / & grand-herb. & géophyte & 3 \\
\hline Anthriscus sylvestris (L.) Hoffm. & Cerfeuil des bois & / & fourré-lis. & hémicrypto. & 6 \\
\hline Argentina anserina (L.) Rydb. & Potentille des oies & / & prairie & hémicrypto. & 4 \\
\hline Bellis perennis L. & Pâquerette & / & prairie & hémicrypto. & 4 \\
\hline Butomus umbellatus L. & Butome en ombelle & / & grand-herb. & géophyte & 4 \\
\hline Callitriche L. & Callitriche & / & aquatique & hémicrypto. & 2 \\
\hline $\begin{array}{l}\text { Capsella bursa-pastoris subsp. rubella } \\
\text { (Reut.) Hobk. }\end{array}$ & Bourse-à-pasteur rougeâtre & / & pelouse & annuelle & 2 \\
\hline Centaurea jacea Gr. & / & / & prairie & hémicrypto. & 1 \\
\hline Cerastium fontanum Baumg. & Céraiste commune & / & culture & hémicrypto. & 2 \\
\hline $\begin{array}{l}\text { Chenopodiastrum hybridum (L.) } \\
\text { S.Fuentes, Uotila \& Borsch }\end{array}$ & Chénopode à feuilles de Stramoine & / & / & annuelle & 1 \\
\hline Cichorium intybus L. & Chicorée sauvage & / & friche & hémicrypto. & 1 \\
\hline Cyclamen hederifolium Aiton & Cyclamen à feuilles de lierre & exogène & forêt & géophyte & 1 \\
\hline Epilobium parviflorum Schreb. & Épilobe à petites fleurs & l & prairie & hémicrypto. & 1 \\
\hline Epipactis helleborine (L.) Crantz & Épipactis à larges feuilles & / & forêt & géophyte & 3 \\
\hline Galium verum $\mathrm{L}$. & Gaillet jaune & / & pelouse & hémicrypto. & 4 \\
\hline Genista tinctoria L. & Genêt des teinturiers & / & fourré-lis. & ligneux & 3 \\
\hline Geranium lucidum L. & Géranium luisant & / & fourré-lis. & annuelle & 8 \\
\hline Geranium pusillum L. & Géranium fluet & / & culture & annuelle & 1 \\
\hline Glyceria maxima (Hartm.) Holmb. & Glycérie aquatique & / & grand-herb. & hémicrypto. & 4 \\
\hline Hordeum murinum L. & Orge sauvage & / & grève & annuelle & 3 \\
\hline Hylotelephium telephium (L.) H.Ohba, & Herbe de saint Jean & / & pelouse & hémicrypto. & 1 \\
\hline Inula britannica L. & Inule des fleuves & LR VU & grand-herb. & hémicrypto. & 2 \\
\hline Juncus tenuis Willd. & Jonc grêle & exogène & prairie & hémicrypto. & 3 \\
\hline Lamium album L. & Lamier blanc & l & fourré-lis. & hémicrypto. & 3 \\
\hline Matricaria discoidea DC. & Matricaire fausse-camomille & exogène & culture & annuelle & 4 \\
\hline Medicago arabica (L.) Huds. & Luzerne tachetée & l & culture & annuelle & 5 \\
\hline Medicago polymorpha L. & Luzerne polymorphe & / & pelouse & annuelle & 1 \\
\hline Medicago sativa subsp. sativa L. & Luzerne cultivée & exogène & friche & hémicrypto. & 5 \\
\hline Mentha aquatica L. & Menthe aquatique & l & grand-herb. & géophyte & 2 \\
\hline Muscari comosum (L.) Mill. & Muscari à toupet & / & culture & géophyte & 1 \\
\hline Phleum pratense L. & Fléole des prés & / & pelouse & hémicrypto. & 1 \\
\hline Plantago coronopus L. & Plantain corne-de-cerf & / & pelouse & bisannuelle & 1 \\
\hline Potamogeton perfoliatus L. & Potamot à feuilles perfoliées & ZNIEFF & aquatique & géophyte & 1 \\
\hline Potentilla argentea L. & Potentille argentée & / & pelouse & hémicrypto. & 3 \\
\hline Prunus cerasifera Ehrh. & Prunier myrobolan & exogène & fourré-lis. & ligneux & 5 \\
\hline Ranunculus bulbosus L. & Renoncule bulbeuse & l & pelouse & géophyte & 2 \\
\hline Reseda luteola L. & Réséda jaunâtre & / & friche & bisannuelle & 1 \\
\hline Rhinanthus minor L. & Petit cocriste & / & pelouse & annuelle & 1 \\
\hline Rosa rugosa Thunb. & Rosier rugueux & exogène & fourré-lis. & ligneux & 2 \\
\hline Sagina apetala Ard. & Sagine apétale & l & culture & annuelle & 1 \\
\hline Salix cinerea L. & Saule cendré & / & fourré-lis. & ligneux & 4 \\
\hline Salix fragilis L. & Saule fragile & / & forêt & ligneux & 4 \\
\hline Salix triandra L. & Saule à trois étamines & / & fourré-lis. & ligneux & 3 \\
\hline Salix viminalis $\mathrm{L}$. & Osier blanc & / & fourré-lis. & ligneux & 8 \\
\hline Stachys sylvatica L. & Épiaire des bois & / & forêt & hémicrypto. & 2 \\
\hline Thalictrum flavum L. & Pigamon jaune & Prot. RC & grand-herb. & hémicrypto. & 3 \\
\hline Trifolium incarnatum L. & Trèfle incarnat & / & j & annuelle & 1 \\
\hline Turritis glabra L. & Arabette glabre & / & fourré-lis. & hémicrypto. & 2 \\
\hline Veronica persica Poir. & Véronique de Perse & exogène & culture & annuelle & 1 \\
\hline
\end{tabular}


ANNEXE 2. - Liste et caractéristiques des espèces originales des îlots par rapport à la berge. Patrimonialité/banalité: EN, en danger; Inv. BLB, invasive pour bassin Loire-Bretagne; Inv. CVL, invasive pour Centre-Val de Loire; LR, liste rouge en région Centre; NT, quasi menacée; Prot. N, protégée en France; Prot. RC, protégée en région Centre-Val de Loire; VU, vulnérable; ZNIEFF, espèce déterminante ZNIEFF. Habitat: fourré-lis., fourrés et lisières; grand-herb., grandes herbes. Type biologique: hémicrypto., hémicryptophyte.

\begin{tabular}{|c|c|c|c|c|c|c|}
\hline \multirow{2}{*}{$\begin{array}{l}\text { Nom latin } \\
\text { TAXREF v11.0 }\end{array}$} & \multirow[b]{2}{*}{ Nom français } & \multirow{2}{*}{$\begin{array}{l}\text { Patrimonialité/ } \\
\text { banalité }\end{array}$} & \multirow{2}{*}{ Habitat } & \multirow[b]{2}{*}{ Type biologique } & \multicolumn{2}{|c|}{ Cumul codes abondance } \\
\hline & & & & & B-nord & B-sud \\
\hline Ajuga genevensis L. & Bugle de Genève & / & pelouse & hémicrypto. & 0 & 1 \\
\hline Alisma plantago-aquatica L. & Grand plantain d'eau & / & grand-herb. & hémicrypto. & 1 & 1 \\
\hline Andryala integrifolia L. & Andryale à feuilles entières & / & culture & bisannuelle & 5 & 4 \\
\hline $\begin{array}{l}\text { Anisantha diandra (Roth) Tutin } \\
\text { ex Tzvelev }\end{array}$ & Brome à deux étamines & / & grève & annuelle & 1 & 0 \\
\hline Anisantha tectorum (L.) Nevski & Brome des toits & / & grève & annuelle & 4 & 2 \\
\hline Anthriscus caucalis M. Bieb. & $\begin{array}{l}\text { Cerfeuil vulgaire à fruits } \\
\text { glabres }\end{array}$ & / & fourré-lis. & annuelle & 1 & 0 \\
\hline Apera spica-venti (L.) P. Beauv. & Jouet-du-Vent & / & / & annuelle & 2 & 2 \\
\hline Arenaria serpyllifolia L. & Sabline à feuilles de serpolet & / & culture & bisannuelle & 9 & 2 \\
\hline Artemisia biennis Willd. & Armoise bisannuelle & exogène & grève & bisannuelle & 3 & 0 \\
\hline Avenella flexuosa (L.) Drejer & Foin tortueux & / & fourré-lis. & hémicrypto. & 1 & 0 \\
\hline Ballota nigra L. & Ballote noire & / & friche & hémicrypto. & 5 & 0 \\
\hline Berberis aquifolium Pursh & Faux Houx & Inv. CVL & fourré-lis. & ligneux & 8 & 0 \\
\hline $\begin{array}{l}\text { Brachypodium rupestre (Host) } \\
\text { Roem. \& Schult. }\end{array}$ & Brachypode des rochers & / & fourré-lis. & hémicrypto. & 0 & 1 \\
\hline Brassica napus L. & Colza & exogène & / & bisannuelle & 1 & 0 \\
\hline Carex leersii F.W.Schultz & Laîche de Leers & / & fourré-lis. & hémicrypto. & 0 & 1 \\
\hline Carex riparia Curtis & Laîche des rives & / & grand-herb. & géophyte & 5 & 0 \\
\hline Centaurea stoebe L. & Centaurée maculée rhénane & LR EN & pelouse & bisannuelle & 2 & 0 \\
\hline Cerastium semidecandrum L. & Céraiste à 5 étamines & / & pelouse & annuelle & 0 & 1 \\
\hline $\begin{array}{l}\text { Chaenorrhinum minus (L.) } \\
\text { Lange }\end{array}$ & Petite linaire & / & culture & annuelle & 1 & 2 \\
\hline Cornus sanguinea $\mathrm{L}$. & Cornouiller sanguin & / & forêt & ligneux & 1 & 0 \\
\hline $\begin{array}{l}\text { Corynephorus canescens (L.) } \\
\text { P.Beauv. }\end{array}$ & Corynéphore blanchâtre & ZNIEFF & pelouse & hémicrypto. & 1 & 0 \\
\hline Cotoneaster Medik. & Cotoneaster & exogène & fourré-lis. & ligneux & 1 & 0 \\
\hline Crepis foetida $\mathrm{L}$. & Crépide fétide & LR EN & pelouse & annuelle & 4 & 1 \\
\hline Cynoglossum officinale L. & Cynoglosse officinale & LR NT & friche & bisannuelle & 6 & 5 \\
\hline Cyperus eragrostis Lam. & Souchet vigoureux & exogène & grand-herb. & hémicrypto. & 1 & 4 \\
\hline Cytisus scoparius (L.) Link & Genêt à balai & / & fourré-lis. & ligneux & 7 & 6 \\
\hline Dipsacus pilosus L. & Cardère poilu & / & forêt & bisannuelle & 0 & 4 \\
\hline $\begin{array}{l}\text { Dysphania botrys (L.) Mosyakin } \\
\text { \& Clemants }\end{array}$ & Chénopode à grappes & / & grève & annuelle & 6 & 4 \\
\hline Echium vulgare L. & Vipérine commune & / & friche & bisannuelle & 9 & 6 \\
\hline Eragrostis pilosa (L.) P.Beauv. & Éragrostis poilu & / & / & annuelle & 0 & 1 \\
\hline Erodium cicutarium (L.) L'Hér. & Érodium à feuilles de cigue & / & culture & annuelle & 4 & 6 \\
\hline Ervum tetraspermum L. & Lentillon & / & / & annuelle & 1 & 0 \\
\hline Erysimum cheiranthoides L. & Vélar fausse-giroflée & / & grève & annuelle & 2 & 3 \\
\hline Euonymus europaeus L. & Bonnet-d'évêque & / & forêt & ligneux & 9 & 3 \\
\hline Eupatorium cannabinum L. & Eupatoire à feuilles de chanvre & e/ & grand-herb. & hémicrypto. & 2 & 3 \\
\hline Filago arvensis L. & Immortelle des champs & / & pelouse & annuelle & 0 & 1 \\
\hline Filipendula ulmaria (L.) Maxim. & Reine des prés & / & grand-herb. & hémicrypto. & 0 & 1 \\
\hline $\begin{array}{l}\text { Galinsoga quadriradiata Ruiz } \\
\text { \& Pav. }\end{array}$ & Galinsoga cilié & exogène & l & annuelle & 1 & 2 \\
\hline Helianthus tuberosus L. & Topinambour & Inv. CVL-BLB & grand-herb. & géophyte & 0 & 1 \\
\hline Herniaria glabra L. & Herniaire glabre & / & pelouse & bisannuelle & 3 & 4 \\
\hline $\begin{array}{l}\text { Himantoglossum hircinum (L.) } \\
\text { Spreng. }\end{array}$ & Orchis bouc & / & pelouse & géophyte & 3 & 0 \\
\hline Holcus mollis L. & Houlque molle & / & culture & hémicrypto. & 2 & 0 \\
\hline Inula conyza DC. & Inule conyze & / & fourré-lis. & hémicrypto. & 5 & 0 \\
\hline Iris foetidissima L. & Iris fétide & / & forêt & géophyte & 8 & 1 \\
\hline $\begin{array}{l}\text { Jacobaea erucifolia (L.) } \\
\text { G.Gaertn., B.Mey. \& Scherb. }\end{array}$ & $\begin{array}{l}\text { Séneçon à feuilles de } \\
\text { Roquette }\end{array}$ & / & friche & hémicrypto. & 0 & 1 \\
\hline Jasione montana L. & Jasione des montagnes & / & pelouse & bisannuelle & 11 & 6 \\
\hline Kickxia elatine (L.) Dumort. & Linaire élatine & / & culture & annuelle & 2 & 2 \\
\hline Lactuca virosa L. & Laitue vireuse & / & friche & bisannuelle & 6 & 0 \\
\hline $\begin{array}{l}\text { Laphangium luteoalbum (L.) } \\
\text { Tzvelev }\end{array}$ & $\begin{array}{l}\text { Pseudognaphale blanc- } \\
\text { jaunâtre }\end{array}$ & ZNIEFF & grève & annuelle & 1 & 0 \\
\hline Leucanthemum vulgare Gr. & Marguerite & exogène & prairie & hémicrypto. & 0 & 2 \\
\hline Malva moschata L. & Mauve musquée & / & prairie & hémicrypto. & 1 & 0 \\
\hline Mentha pulegium L. & Menthe pouliot & / & grand-herb. & hémicrypto. & 0 & 7 \\
\hline Mentha suaveolens Ehrh. & Menthe à feuilles rondes & / & prairie & hémicrypto. & 1 & 1 \\
\hline Micropyrum tenellum (L.) Link & Catapode des graviers & LR NT & pelouse & annuelle & 3 & 6 \\
\hline Moehringia trinervia (L.) Clairv. & Sabline à trois nervures & / & forêt & annuelle & 6 & 3 \\
\hline Myosotis arvensis (L.) Hill & Myosotis des champs & / & culture & annuelle & 7 & 2 \\
\hline
\end{tabular}


ANNEXE 2. - Suite.

\begin{tabular}{|c|c|c|c|c|c|c|}
\hline \multirow{2}{*}{$\begin{array}{l}\text { Nom latin } \\
\text { TAXREF v11.0 }\end{array}$} & \multirow[b]{2}{*}{ Nom français } & \multirow{2}{*}{$\begin{array}{l}\text { Patrimonialité/ } \\
\text { banalité }\end{array}$} & \multirow{2}{*}{ Habitat } & \multirow[b]{2}{*}{ Type biologique } & \multicolumn{2}{|c|}{ Cumul codes abondance } \\
\hline & & & & & B-nord & B-sud \\
\hline Myosotis discolor Gr. & Myosotis discolore & / & / & annuelle & 1 & 0 \\
\hline Myriophyllum spicatum L. & Myriophylle à épis & / & aquatique & géophyte & 1 & 5 \\
\hline Ononis spinosa L. & Bugrane épineuse & / & pelouse & hémicrypto. & 0 & 1 \\
\hline Oxalis dillenii Jacq. & Oxalis dressé & exogène & friche & hémicrypto. & 1 & 1 \\
\hline $\begin{array}{l}\text { Panicum dichotomiflorum } \\
\text { Michx. }\end{array}$ & Panic à fleurs dichotomes & exogène & / & annuelle & 0 & 2 \\
\hline Parietaria judaica L. & Pariétaire des murs & / & / & hémicrypto. & 5 & 0 \\
\hline $\begin{array}{l}\text { Parthenocissus inserta } \\
\text { (A.Kern.) Fritsch }\end{array}$ & Vigne-vierge commune & Inv. CVL-BLB & / & ligneux & 8 & 10 \\
\hline Pastinaca sativa L. & Panais cultivé & / & friche & bisannuelle & 0 & 1 \\
\hline $\begin{array}{l}\text { Petrorhagia prolifera (L.) } \\
\text { P.W.Ball \& Heywood }\end{array}$ & Oeillet prolifère & / & pelouse & annuelle & 6 & 0 \\
\hline Phleum phleoides (L.) H.Karst. & Fléole de Boehmer & ZNIEFF & pelouse & hémicrypto. & 0 & 1 \\
\hline $\begin{array}{l}\text { Pilosella officinarum } \\
\text { F.W.Schultz \& Sch.Bip. }\end{array}$ & Piloselle & / & pelouse & hémicrypto. & 6 & 1 \\
\hline $\begin{array}{l}\text { Plantago arenaria Waldst. \& } \\
\text { Kit. }\end{array}$ & Plantain scabre & / & grève & annuelle & 12 & 11 \\
\hline Poa compressa L. & Pâturin comprimé & / & pelouse & géophyte & 1 & 0 \\
\hline Poa pratensis L. & Pâturin des prés & / & prairie & géophyte & 5 & 3 \\
\hline $\begin{array}{l}\text { Potamogeton berchtoldii } \\
\text { Fieber }\end{array}$ & Potamot de Berchtold & / & aquatique & annuelle & 0 & 1 \\
\hline Potamogeton crispus L. & Potamot crépu & / & aquatique & géophyte & 0 & 1 \\
\hline Potentilla verna L. & $\begin{array}{l}\text { Potentille de } \\
\text { Tabernaemontanus }\end{array}$ & / & pelouse & hémicrypto. & 1 & 0 \\
\hline Poterium sanguisorba L. & Pimprenelle à fruits réticulés & / & pelouse & hémicrypto. & 4 & 0 \\
\hline Primula veris $\mathrm{L}$. & Coucou & / & pelouse & hémicrypto. & 0 & 2 \\
\hline Quercus rubra L. & Chêne rouge d'Amérique & exogène & forêt & ligneux & 3 & 0 \\
\hline Ranunculus acris L. & Bouton d'or & / & prairie & hémicrypto. & 0 & 3 \\
\hline Ribes rubrum L. & Groseillier rouge & / & forêt & ligneux & 6 & 1 \\
\hline Rumex acetosella L. & Petite oseille & / & culture & hémicrypto. & 4 & 1 \\
\hline Rumex maritimus L. & Patience maritime & / & grève & bisannuelle & 1 & 2 \\
\hline Ruscus aculeatus L. & Fragon & / & forêt & ligneux & 4 & 0 \\
\hline Scabiosa columbaria L. & Scabieuse colombaire & / & pelouse & hémicrypto. & 1 & 0 \\
\hline Scleranthus annuus L. & Gnavelle annuelle & / & / & annuelle & 0 & 1 \\
\hline Scrophularia canina L. & Scrofulaire des chiens & ZNIEFF & / & hémicrypto. & 2 & 0 \\
\hline Sedum acre L. & Poivre de muraille & / & pelouse & hémicrypto. & 10 & 9 \\
\hline Sedum rupestre L. & Orpin réfléchi & / & pelouse & hémicrypto. & 4 & 3 \\
\hline Sedum sexangulare $\mathrm{L}$. & Orpin de Bologne & LR VU & pelouse & hémicrypto. & 1 & 0 \\
\hline Senecio inaequidens DC. & Séneçon sud-africain & Inv. BLB & friche & hémicrypto. & 3 & 3 \\
\hline Senecio viscosus L. & Séneçon visqueux & / & / & annuelle & 2 & 0 \\
\hline $\begin{array}{l}\text { Setaria italica subsp. viridis } \\
\text { (L.) Thell. }\end{array}$ & Sétaire verte & / & culture & annuelle & 6 & 3 \\
\hline $\begin{array}{l}\text { Setaria pumila (Poir.) Roem. } \\
\quad \& \text { Schult. }\end{array}$ & Sétaire glauque & / & / & annuelle & 2 & 3 \\
\hline Thymus pulegioides L. & Thym commun & / & pelouse & hémicrypto. & 0 & 1 \\
\hline Torilis arvensis (Huds.) Link & Torilis des champs & / & / & annuelle & 5 & 1 \\
\hline Tragopogon dubius Scop. & Grand salsifis & / & friche & hémicrypto. & 6 & 3 \\
\hline Trifolium arvense L. & Trèfle des champs & / & pelouse & bisannuelle & 3 & 0 \\
\hline Triticum L. & Blé & exogène & / & annuelle & 2 & 1 \\
\hline Vallisneria spiralis L. & Vallisnérie en spirale & exogène & aquatique & hémicrypto. & 1 & 1 \\
\hline Verbascum thapsus L. & Molène bouillon-blanc & / & friche & bisannuelle & 1 & 0 \\
\hline Verbena bonariensis L. & Verveine & exogène & friche & hémicrypto. & 1 & 0 \\
\hline Veronica chamaedrys L. & Véronique petit chêne & / & fourré-lis. & hémicrypto. & 0 & 2 \\
\hline Veronica officinalis L. & Véronique officinale & / & fourré-lis. & hémicrypto. & 0 & 1 \\
\hline Viola riviniana Rchb. & Violette de Rivinus & / & forêt & hémicrypto. & 0 & 1 \\
\hline Vulpia ciliata Dumort. & Vulpie ambiguë & / & pelouse & annuelle & 2 & 3 \\
\hline Zannichellia palustris L. & Zannichellie des marais & ZNIEFF & aquatique & géophyte & 0 & 1 \\
\hline
\end{tabular}


ANNEXE 3. - Liste et caractéristiques des espèces préférentielles de la berge par rapport aux îlots B-nord et B-sud agrégés (moyenne du cumul des cotations d'abondance). Patrimonialité/banalité: ZNIEFF, espèce déterminante ZNIEFF. Habitat: fourré-lis., fourrés et lisières; grand-herb., grandes herbes. Type biologique: hémicrypto., hémicryptophyte. En gris, espèces à différentiel marqué (écart de cumul des codes d'abondance $\geq 7$ )

\begin{tabular}{|c|c|c|c|c|c|c|}
\hline \multirow[b]{2}{*}{ Nom latin TAXREF v11.0 } & \multirow[b]{2}{*}{ Nom français } & \multirow[b]{2}{*}{$\begin{array}{l}\text { Patrimonialité/ } \\
\text { banalité }\end{array}$} & \multirow[b]{2}{*}{ Habitat } & \multirow[b]{2}{*}{ Type biologique } & \multicolumn{2}{|c|}{ Cumul codes abondance } \\
\hline & & & & & Berge & $\begin{array}{c}\text { B-nord \& } \\
\text { B-sud }\end{array}$ \\
\hline Allium vineale $\mathrm{L}$. & Ail des vignes & / & culture & géophyte & 5 & 0,0 \\
\hline Anthriscus sylvestris (L.) Hoffm. & Cerfeuil des bois & / & fourré-lis. & hémicrypto. & 6 & 0,0 \\
\hline Argentina anserina (L.) Rydb. & Potentille des oies & / & prairie & hémicrypto. & 4 & 0,0 \\
\hline Astragalus glycyphyllos L. & Réglisse sauvage & / & fourré-lis. & hémicrypto. & 7 & 1,5 \\
\hline Bellis perennis L. & Pâquerette & / & prairie & hémicrypto. & 4 & 0,0 \\
\hline Butomus umbellatus L. & Butome en ombelle & / & grand-herb. & géophyte & 4 & 0,0 \\
\hline Cirsium vulgare (Savi) Ten. & Cirse commun & / & friche & bisannuelle & 5 & 1,0 \\
\hline Galium elongatum C.Presl & Gaillet allongé & / & grand-herb. & hémicrypto. & 7 & 2,0 \\
\hline Galium verum $\mathrm{L}$. & Gaillet jaune & l & pelouse & hémicrypto. & 4 & 0,0 \\
\hline Geranium lucidum L. & Géranium luisant & l & fourré-lis. & annuelle & 8 & 0,0 \\
\hline Glyceria maxima (Hartm.) Holmb. & Glycérie aquatique & / & grand-herb. & hémicrypto. & 4 & 0,0 \\
\hline Iris pseudacorus L. & Iris faux acore & / & grand-herb. & géophyte & 9 & 2,5 \\
\hline Juncus compressus Jacq. & $\begin{array}{l}\text { Jonc à tiges } \\
\text { comprimées }\end{array}$ & / & prairie & géophyte & 5 & 0,5 \\
\hline Lolium perenne L. & Ivraie vivace & / & prairie & hémicrypto. & 13 & 0,5 \\
\hline Matricaria discoidea DC. & $\begin{array}{l}\text { Matricaire fausse- } \\
\text { camomille }\end{array}$ & exogène & culture & annuelle & 4 & 0,0 \\
\hline Medicago arabica (L.) Huds. & Luzerne tachetée & / & culture & annuelle & 5 & 0,0 \\
\hline Medicago sativa subsp. sativa L. & Luzerne cultivée & exogène & friche & hémicrypto. & 5 & 0,0 \\
\hline $\begin{array}{l}\text { Medicago sativa subsp. ambigua } \\
\text { (Trautv.) Tutin }\end{array}$ & Luzerne changeante & / & friche & hémicrypto. & 6 & 0,5 \\
\hline Myosotis scorpioides L. & Myosotis des marais & l & grand-herb. & hémicrypto. & 11 & 1,5 \\
\hline Persicaria amphibia (L.) Gray & Persicaire flottante & / & culture & géophyte & 10 & 4,0 \\
\hline $\begin{array}{l}\text { Phragmites australis (Cav.) Trin. } \\
\quad \text { ex Steud. }\end{array}$ & Roseau & / & grand-herb. & géophyte & 9 & 3,5 \\
\hline Poa annua L. & Pâturin annuel & / & culture & bisannuelle & 11 & 5,5 \\
\hline Potentilla reptans L. & Potentille rampante & / & culture & hémicrypto. & 9 & 1,0 \\
\hline Prunus cerasifera Ehrh. & Prunier myrobolan & exogène & fourré-lis. & ligneux & 5 & 0,0 \\
\hline Prunus mahaleb L. & Bois de Sainte-Lucie & exogène & fourré-lis. & ligneux & 8 & 4,0 \\
\hline Rumex crispus L. & Rumex crépu & / & culture & hémicrypto. & 7 & 2,5 \\
\hline Salix alba L. & Saule blanc & / & forêt & ligneux & 16 & 5,5 \\
\hline Salix cinerea L. & Saule cendré & / & fourré-lis. & ligneux & 4 & 0,0 \\
\hline Salix fragilis L. & Saule fragile & / & forêt & ligneux & 4 & 0,0 \\
\hline Salix viminalis $\mathrm{L}$. & Osier blanc & / & fourré-lis. & ligneux & 8 & 0,0 \\
\hline Salix x rubens Schrank & Osier jaune & / & forêt & ligneux & 6 & 1,5 \\
\hline Sisymbrium officinale (L.) Scop. & Moutarde & / & grève & bisannuelle & 6 & 1,0 \\
\hline Tordylium maximum L. & Tordyle majeur & / & friche & bisannuelle & 5 & 0,5 \\
\hline Trifolium repens L. & Trèfle rampant & / & prairie & hémicrypto. & 8 & 2,0 \\
\hline Ulmus laevis Pall. & Orme lisse & ZNIEFF & forêt & ligneux & 8 & 3,0 \\
\hline Vicia segetalis Thuill. & Vesce des moissons & / & culture & annuelle & 7 & 0,5 \\
\hline
\end{tabular}


ANNEXE 4. - : Liste et caractéristiques des espèces préférentielles des îlots B-nord et B-sud agrégés par rapport à la berge. Patrimonialité/banalité: Inv. BLB, invasive pour bassin Loire-Bretagne; Inv. CVL, invasive pour Centre-Val de Loire; LR, liste rouge en région Centre; NT, quasi menacée. Habitat: grand-herb., grandes herbes; fourré-lis., fourrés et lisières. Type biologique: hémicrypto., hémicryptophyte. En gris, espèces à différentiel marqué (écart de cumul des codes d'abondance $\geq 7$ ).

\begin{tabular}{|c|c|c|c|c|c|c|}
\hline \multirow[b]{2}{*}{$\begin{array}{l}\text { Nom latin } \\
\text { TAXREF v11.0 }\end{array}$} & \multirow[b]{2}{*}{ Nom français } & & \multirow[b]{2}{*}{ Type biologique } & \multicolumn{2}{|c|}{ Cumul codes abondance } \\
\hline & & $\begin{array}{l}\text { Patrimonialité/ } \\
\text { banalité }\end{array}$ & Habitat & & Berge & $\begin{array}{c}\text { B-nord \& } \\
\text { B-sud }\end{array}$ \\
\hline $\begin{array}{l}\text { Aethusa cynapium subsp. elata } \\
\text { (Friedl.) Schübl. \& G.Martens }\end{array}$ & Fausse petite Ciguë & / & forêt & bisannuelle & 3 & 7,0 \\
\hline Aira caryophyllea L. & Canche caryophillée & / & pelouse & annuelle & 1 & 6,0 \\
\hline Amaranthus hybridus L. & Amarante hybride & exogène & culture & annuelle & 3 & 8,5 \\
\hline Andryala integrifolia L. & Andryale à feuilles entières & / & culture & bisannuelle & 0 & 4,5 \\
\hline Arenaria serpyllifolia L. & Sabline à feuilles de serpolet & / & culture & bisannuelle & 0 & 5,5 \\
\hline Berberis aquifolium Pursh & Faux Houx & Inv. CVL & fourré-lis. & ligneux & 0 & 4,0 \\
\hline Carex spicata Huds. & Laîche en épis & / & fourré-lis. & hémicrypto. & 1 & 5,5 \\
\hline Chondrilla juncea L. & Chondrille à tige de jonc & / & friche & bisannuelle & 2 & 10,0 \\
\hline Corrigiola littoralis $\mathrm{L}$. & Corrigiole des rivages & / & grève & annuelle & 7 & 15,0 \\
\hline Crepis capillaris (L.) Wallr. & Crépide capillaire & 1 & culture & bisannuelle & 2 & 11,0 \\
\hline Cynoglossum officinale L. & Cynoglosse officinale & LR NT & friche & bisannuelle & 0 & 5,5 \\
\hline $\begin{array}{l}\text { Cyperus esculentus var. } \\
\text { leptostachyus Boeckeler }\end{array}$ & Souchet comestible & exogène & grève & géophyte & 1 & 9,5 \\
\hline Cytisus scoparius (L.) Link & Genêt à balai & / & fourré-lis. & ligneux & 0 & 6,5 \\
\hline Digitaria sanguinalis (L.) Scop. & Digitaire sanguine & l & culture & annuelle & 1 & 10,0 \\
\hline $\begin{array}{l}\text { Dysphania botrys (L.) Mosyakin \& } \\
\text { Clemants }\end{array}$ & Chénopode à grappes & / & grève & annuelle & 0 & 5,0 \\
\hline Echium vulgare L. & Vipérine commune & / & friche & bisannuelle & 0 & 7,5 \\
\hline $\begin{array}{l}\text { Eragrostis pectinacea (Michx.) } \\
\text { Nees }\end{array}$ & Éragrostis en peigne & Inv. BLB & grève & annuelle & 4 & 14,0 \\
\hline Erigeron annuus (L.) Desf. & Vergerette annuelle & exogène & grand-herb. & hémicrypto. & 1 & 5,5 \\
\hline Erodium cicutarium (L.) L'Hér. & Érodium à feuilles de cigue & / & culture & annuelle & 0 & 5,0 \\
\hline Euonymus europaeus L. & Bonnet-d'évêque & / & forêt & ligneux & 0 & 6,0 \\
\hline Iris foetidissima $\mathrm{L}$. & Iris fétide & / & forêt & géophyte & 0 & 4,5 \\
\hline Jasione montana L. & Jasione des montagnes & / & pelouse & bisannuelle & 0 & 8,5 \\
\hline Lepidium virginicum L. & Passerage de Virginie & exogène & grève & bisannuelle & 3 & 10,5 \\
\hline Ligustrum vulgare L. & Troëne & / & fourré-lis. & ligneux & 1 & 6,5 \\
\hline Lindernia dubia (L.) Pennell & Lindernie fausse-gratiole & Inv. BLB & grève & annuelle & 3 & 8,5 \\
\hline Micropyrum tenellum (L.) Link & Catapode des graviers & LR NT & pelouse & annuelle & 0 & 4,5 \\
\hline Moehringia trinervia (L.) Clairv. & Sabline à trois nervures & / & forêt & annuelle & 0 & 4,5 \\
\hline Myosotis arvensis (L.) Hill & Myosotis des champs & / & culture & annuelle & 0 & 4,5 \\
\hline $\begin{array}{l}\text { Parthenocissus inserta (A.Kern.) } \\
\text { Fritsch }\end{array}$ & Vigne-vierge commune & Inv. CVL-BLB & l & ligneux & 0 & 9,0 \\
\hline Plantago arenaria Waldst. \& Kit. & Plantain scabre & / & grève & annuelle & 0 & 11,5 \\
\hline Poa pratensis L. & Pâturin des prés & / & prairie & géophyte & 0 & 4,0 \\
\hline Portulaca oleracea L. & Pourpier potager & / & culture & annuelle & 6 & 12,0 \\
\hline Rhamnus cathartica L. & Nerprun purgatif & / & forêt & ligneux & 1 & 8,0 \\
\hline Sedum acre L. & Poivre de muraille & / & pelouse & hémicrypto. & 0 & 9,5 \\
\hline Sedum album $\mathrm{L}$. & Orpin blanc & l & pelouse & hémicrypto. & 1 & 11,0 \\
\hline Sedum rubens $\mathrm{L}$. & Orpin rougeâtre & / & fourré-lis. & annuelle & 1 & 6,0 \\
\hline $\begin{array}{l}\text { Setaria italica subsp. viridis (L.) } \\
\text { Thell. }\end{array}$ & Sétaire verte & / & culture & annuelle & 0 & 4,5 \\
\hline Spergula rubra (L.) D.Dietr. & Sabline rouge & / & pelouse & bisannuelle & 2 & 6,5 \\
\hline Tragopogon dubius Scop. & Grand salsifis & / & friche & hémicrypto. & 0 & 4,5 \\
\hline Verbascum densiflorum Bertol. & Molène faux-bouillon-blanc & / & friche & bisannuelle & 2 & 7,0 \\
\hline Vulpia bromoides (L.) Gray & Vulpie queue-d'écureuil & / & pelouse & annuelle & 1 & 6,0 \\
\hline Vulpia myuros (L.) C.C.Gmel. & Vulpie queue-de-rat & / & pelouse & annuelle & 1 & 10,5 \\
\hline
\end{tabular}

